\title{
Coupling of acoustic and intrinsic modes in 1-D combustor models
}

\author{
Nalini Kanta Mukherjee, Victor Shrira \\ School of Computing and Mathematics, Keele University, ST55BG, UK \\ e-mail: nalinikanta65@gmail.com, n.k.mukherjee@keele.ac.uk
}

The work is concerned with the theoretical examination of a new type of thermo-acoustic instability in combustors not reported in the literature. The instability results from linear coupling between the conventional acoustic mode and the recently discovered 'flame intrinsic modes' (Hoeijmakers et al., 2014). Within the framework of a 1-D model of a quarter wave resonator with the standard $n-\tau$ model of flame heat release, intrinsic-acoustic mode coupling occurs when the real parts of the frequencies of neighboring acoustic and flame intrinsic modes at small interaction index $n$ are close. While at small $n$ the eigen-functions of close acoustic and flame intrinsic modes clearly exhibit their distinctive identities, with increase of $n$ the mode identities become blurred and the eigen-functions of acoustic modes resemble more and more those of flame intrinsic modes and at a certain $n$ become indistinguishable. We refer them as coupled intrinsic-acoustic modes or coupled modes. When the 'Rayleigh index' for a coupled mode behaving as an acoustic mode at small $n$ is negative, at a larger $n$ such a mode can nevertheless become unstable at one of the nearby intrinsic mode frequencies. We find analytically the instability domain due to coupling in the parameter space. Near the instability boundary, we reduce the transcendental dispersion relation to a quadratic or, if higher accuracy is desired, to a quartic equation. These models capture well all four possible coupling scenarios.

Keywords: Thermo-acoustic instabilities, intrinsic-acoustic mode coupling, flame intrinsic modes, $n-\tau$ model, combustors.

\section{Introduction}

Combustion driven oscillations, also known as 'combustion instabilities', have been a major source of concern for the design of low emissions gas turbine (Lieuwen and Yang, 2005). In general, in a combustor both the conventional acoustic and recently discovered flame intrinsic modes (Hoeijmakers et al., 2014) (for brevity we refer the latter as intrinsic modes) can become unstable. This leads to the following two qualitatively different scenarios of instability in the simplest models of closed-open combustor with ideal end conditions (assuming the $n-\tau$ model of flame heat release (Crocco and Cheng, 1956)), (i) Acoustic modes are becoming unstable at zero threshold, $n_{t h}^{a}=0$, with the instability frequency $\omega_{0}^{a}=\left(2 m^{a}+1\right)(\pi c / 2 L)$ for a quarter-wave resonator (Munjal, 1987), (where the mode number $m^{a}=0,1,2 \ldots$, while $L$ is the length of the resonator and $c$ is the speed of sound); and (ii) Intrinsic modes are becoming unstable at $n_{t h}^{i}$ with the instability frequency given by Mukherjee and Shrira (2017). The first type of instability was in the focus of combustion research for the past few decades (e.g. Lieuwen and Yang, 2005). The second type of instability, is that of an isolated intrinsic mode, that is not coupled to an acoustic mode. In the first type of instability due to acoustic modes, 
the flame heat release rate and the acoustic pressure are in-phase with each other, implying positivity of the 'Rayleigh Index', $R_{a}$ (given as $R_{a}=\int_{0}^{T} \int_{0}^{V} p^{\prime}(x, t) q^{\prime}(x, t) d v d t$, where $p^{\prime}$ is the acoustic pressure oscillation, $T$ is the time period, $V$ is combustor volume (control volume), $q^{\prime}$ is heat release oscillation). When the flame heat release rate and acoustic pressure are out of phase with each other, the Rayleigh index is negative, which ensures the acoustic mode decay and, hence, the first type of instability does not occur. However, such an acoustic mode might nevertheless become unstable due to coupling with one of the intrinsic modes in its vicinity in the frequency domain. This work is focused on this novel type of combustor instability. It will be shown here that under this scenario, as the acoustic mode approaches the instability boundary it behaves exactly as an intrinsic mode.

The salient features of intrinsic instability of 1-D closed-open combustors based on the $n-\tau$ model of flame heat release were established by Mukherjee and Shrira (2017), where exact analytical expressions for the neutral curve of intrinsic instability and the corresponding frequency of the intrinsic mode on the neutral curve were found. The findings are based on a remarkable phenomenon of 'decoupling' or 'factorization' of the dispersion relation on the neutral curve,

$$
2 \operatorname{in}_{\text {th }}^{i} \sin \left(\omega_{c}^{i} \tau\right) \sin \left(k_{1} x_{q}\right) \sin \left(k_{2}\left(x_{q}-L\right)\right)=0,
$$

where $\omega_{c}^{i}$ is the discrepancy between the real part of the frequency on the neutral curve and the real part of the frequency in the limit of small $n$ (i.e. $\left.\omega_{0}^{i}\right), k_{i}=\omega / c_{i}(i=1,2)$ are the wave numbers across the flame, located at $x=x_{q}$. On the neutral curve the intrinsic modes were found to be completely decoupled from the combustor environment and their frequencies to be governed solely by the time lag of the system. The neutral curves for the intrinsic modes, i.e. the threshold value of $n$ as function of time lag $\tau$, were found to be a combination of segments of three types, $\omega_{c}^{i} \tau=0, \omega_{c}^{i} \tau=\pi$ and $\omega_{c}^{i} \tau=-\pi$ given as,

$$
n_{\text {th }}^{i}=\frac{(\alpha+1) \cos \left\{\left(\omega_{0}^{i}+\omega_{c}^{i}\right) \beta_{1}\right\}+(\alpha-1) \cos \left\{\left(\omega_{0}^{i}+\omega_{c}^{i}\right) \beta_{2}\right\}}{2 \cos \left(\omega_{c}^{i} \tau\right) \sin \left(k_{1} x_{q}\right) \sin \left\{k_{2}\left(x_{q}-L\right)\right\}} .
$$

It was also reported that near the intersection of the different segments, the neutral curves manifest two solutions for a single time lag. They were interpreted as coupled-intrinsic and coupled-acoustic mode, respectively. The analysis in Mukherjee and Shrira (2017) was based on the key assumption that there are distinct separate intrinsic and acoustic modes. This assumption is sometimes too restrictive. In any combustion system under certain conditions, the intrinsic modes and acoustic modes might have close frequencies and thus, might so strongly interact (or couple) with each other that treating these modes separately is no longer possible. To our knowledge, so far, the linear coupling of intrinsic and acoustic modes has not been considered in the literature.

Here, within the framework of 1-D $n-\tau$ models of combustors we, without much loss of generality, consider an example of acoustic-intrinsic mode coupling for a 1-D quarter wave 
resonator (i.e. a closed-open combustor). We stress that whatever might be the specific model of the combustor, there is always existence of mode coupling between the acoustic and the intrinsic modes. The mathematical models of such a coupling might differ from that examined here, and, therefore, should be a subject of dedicated studies.

We show that this coupling is an important element of the big picture of combustor thermoacoustic instabilities, as an otherwise decaying acoustic mode could become unstable due to coupling with an intrinsic mode. We also address an open question raised by Mukherjee and Shrira (2017) about the possible origin of two solutions for a single time lag on the neutral

curves. We show that the neutral curve segments $\omega_{c}^{i} \tau=\pi$ and $\omega_{c}^{i} \tau=-\pi$, are indeed manifestations of intrinsic-acoustic mode coupling in combustors. We explore all possible types of coupling that might exist in combustors. Linear mode coupling has been studied in different research fields, for example, in plasmas, hydrodynamics, physical oceanography (Craik, 1985).

The paper is organized as follows. In $\S \mathbf{2}$ we provide an example of numerical evidence of intrinsic-acoustic mode coupling, using the dispersion relation of a quarter wave resonator. In $\S 3$ we discuss the pressure profiles of the coupled intrinsic and acoustic modes. In $\$ \mathbf{4}$ we derive a simple mathematical model which captures the main features of the intrinsic-acoustic mode coupling. In the simplest model, the full dispersion relation is reduced to a quadratic equation, which is later improved to a quartic equation for better accuracy. The four possible scenarios of coupling are identified. In $\$ \mathbf{5}$ the domain on the neutral curve where intrinsicacoustic mode coupling occurs is identified. In $\$ \mathbf{6}$ we formulate concluding remarks and outline the scope of future work.

\section{Intrinsic-acoustic mode coupling in the complex frequency plane}

Mukherjee and Shrira (2017) employed compact dispersion relation for a 1-D quarter wave resonator (i.e. a closed-open combustor) based on the $n-\tau$ model of flame heat release along with the temperature and cross-section jump across the flame. It was assumed that the flame is compact, i.e. the combustion region of interest has much smaller length scale than the acoustic wavelength, which implies that the distribution of the heat release is not essential; instead the quantity of relevance is the total, spatially integrated value (Lieuwen, 2012).

Fig. 1 presents a schematic sketch of a quarter-wave resonator with a compact heat source at $x=x_{q}$, with $x$ being the longitudinal coordinate with the origin at the closed end of the resonator. The compact flame assumption implies that the flame is presumed to lie on a single axial hypothetical plane and any distribution of flame heat release rate in the axial direction is neglected. $A_{1}, A_{2}$ and $B_{1}, B_{2}$ indicate the pressure amplitudes for the forward going and backward travelling waves in the upstream and the downstream region, respectively. In this model, only 1-D plane wave propagation is considered, while the effects due to the transverse wave propagation are ignored. In actual combustion systems, the temperature is distributed along the length of the combustor. Often this distribution is unsteady, giving rise to entropy waves (e.g. Polifke et al., 2001), an effect which we neglect here. To retain simplicity necessary for the analytical model, the mean temperature is assumed to jump from $T_{1}$ to $T_{2}$ across the flame. The cross-sectional area is also assumed to have a jump across the flame from $S_{1}$ to $S_{2}$ 
. The losses due to damping and heat transfer across the combustor walls are not considered in our model. In practical combustors mean flow is usually small and we ignore the effect of mean flow in our study. Our next assumption is that there is no turbulence or hydrodynamic instability. For simplicity, we also assume the combustion to take place instantaneously at the flame location, $x=x_{q}$.

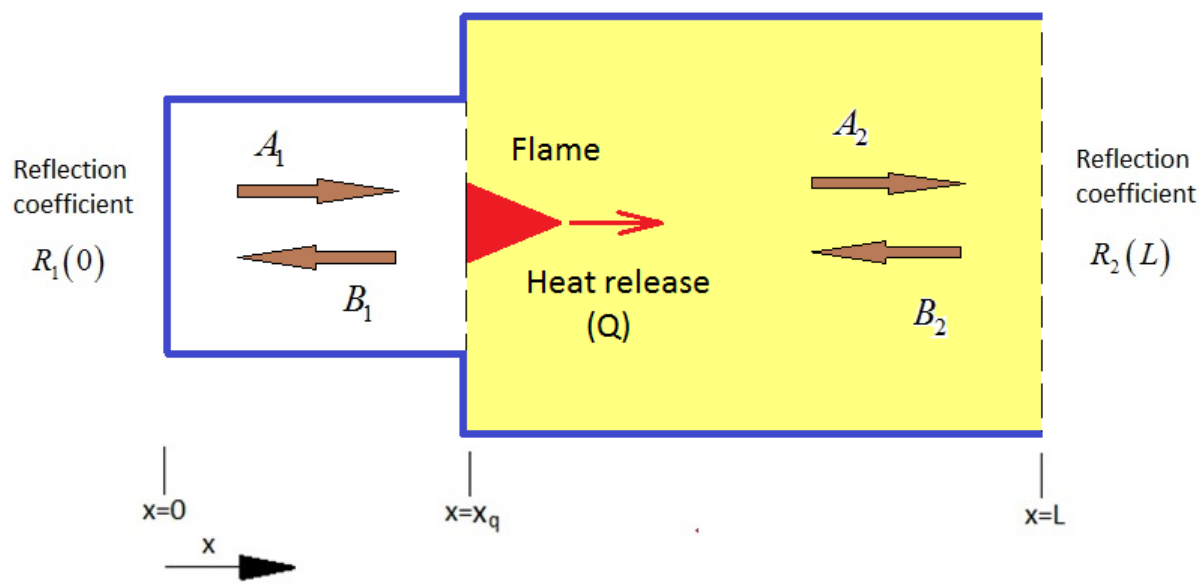

Figure 1: Schematic sketch of a combustor with closed-open end boundary conditions. Thick arrows (brown online) symbolize the forward going and backward travelling waves upstream and downstream of the flame. At the flame location, $x=x_{q}$, the combustor has a temperature, as well as, cross-section jump. Shaded region (yellow online) indicates the domain of higher temperature, once the temperature jump due to combustion takes place.

Assuming plane wave propagation, the acoustic pressure and particle velocity in the upstream and downstream regions of the flame can be written as,

$$
\begin{aligned}
& \tilde{p}_{1}(x, t)=\left[A_{1} e^{i k_{1} x}+B_{1} e^{-i k_{1} x}\right] e^{-i \omega t}, \tilde{u}_{1}(x, t)=\left(\frac{1}{\rho_{1} c_{1}}\right)\left[A_{1} e^{i k_{1} x}-B_{1} e^{-i k_{1} x}\right] e^{-i \omega t}, \text { and } \\
& \tilde{p}_{2}(x, t)=\left[A_{2} e^{i k_{2} x}+B_{2} e^{-i k_{2} x}\right] e^{-i \omega t}, \tilde{u}_{2}(x, t)=\left(\frac{1}{\rho_{2} c_{2}}\right)\left[A_{2} e^{i k_{2} x}-B_{2} e^{-i k_{2} x}\right] e^{-i \omega t},
\end{aligned}
$$

where $k_{i}=\omega / c_{i}(i=1,2)$ are the wave numbers, $\rho_{1}, \rho_{2}$ are the mean densities, $c_{1}, c_{2}$ are the speed of sound upstream and downstream the flame, respectively and $\omega$ is the complex frequency. Ideal boundaries at both ends of the combustor dictate the boundary conditions,

$$
\begin{gathered}
\text { at } x=0, A_{1} / B_{1}=R_{1}(0)=1, \text { and, } \\
\text { at } x=L, B_{2} e^{-i k L} / A_{2} e^{i k L}=R_{2}(L)=-1 .
\end{gathered}
$$

In the current model, the flame is assumed to act like an acoustic monopole with a volume outflow $(\gamma-1)\left(\tilde{Q} / \rho_{1} c_{1}^{2}\right)$, where, $\tilde{Q}(t)$ is the heat release rate at $x=x_{q}$ and $\gamma$ represents the ratio of specific heats of air $\left(c_{p} / c_{v}\right)$ (Dowling, 1995). In line with Mukherjee and Shrira (2017), 
we assume the linear heat release rate of the flame to obey the following equation (see e.g. Truffin and Poinsot, 2005),

$$
\tilde{Q}(t)=\left(\rho_{1} S_{1} c_{1}^{2} /(\gamma-1)\right) n \tilde{u}_{1}(t-\tau) .
$$

where $n$ and $\tau$ are the interaction index and time lag, respectively. The heat release rate fluctuations, $\tilde{Q}$, are assumed to be proportional to the local velocity upstream of the flame, $\tilde{u}_{1}$, with a time lag, $\tau$. This assumption respects causality between local velocity fluctuation upstream of the flame and heat release rate fluctuation at the flame. In other words, the local velocity fluctuation upstream of the flame always leads the heat release rate fluctuation at the flame. In the time domain, this lead is described by the factor $\tau$, known as time lag. In the frequency domain, we can write, $\tilde{Q}(\omega)=\left(\rho_{1} S_{1} c_{1}^{2} /(\gamma-1)\right) n e^{i \omega \tau} \tilde{u}_{1}(\omega)$.

It should be noted that in our present model we assume the 'velocity coupling' mechanism, in which the upstream velocity oscillations lead to the flame heat release oscillations. In a similar manner, we can have 'equivalence ratio coupling' (equivalence ratio is the actual fuel-air ratio/Stoichiometric fuel-air ratio), 'acoustic pressure coupling', as well as, 'entropy coupling' and 'acceleration coupling' (Lieuwen, 2012). The total unsteady heat release in the linear approximation is the superposition of the velocity, pressure and equivalence ratio coupling. In the regimes characterized by low Strouhal number, $S t$ ( $S t=f * l / U$, where, $f$ is frequency in $\mathrm{Hz}, l$ is a characteristic length scale, e.g. the length of flame in the direction of flow, $U$ is axial flow velocity), the equivalence ratio and velocity coupled mechanisms are of similar order for lean flames, whereas, the pressure coupled mechanism contribution is negligible for low Mach number flows. At higher frequencies, pressure coupling might contribute significantly for some cases, as the pressure coupled unsteady heat release rolls off with frequency slower than velocity and fuel/air ratio coupled mechanisms (Lieuwen, 2012).

The $n-\tau$ flame heat release model has its own limitations (see e.g. Lieuwen, 2003, 2012; Magina et al., 2019). The $n-\tau$ heat release model, indeed, is an approximation of the heat release dynamics in the low Strouhal number limit $(S t<<1)$. The time lag $\tau$, is equivalent to replacing the distributed flame by a concentrated source at this location. While the gain $n$, is straightforward, the time lag is a complex function of parameters such as Peclet number $\mathrm{Pe}(\mathrm{Pe}$ is the ratio of the diffusion time scale to the convection time scale) and Stoichiometric mixture fraction for non-premixed flames (Lieuwen, 2003). These parametric dependencies of $\tau$ cannot be calculated analytically but can be extracted from simulations. For premixed flames, the time lag depends on the flame length as well as on the axial flow velocity (Lieuwen, 2003, 2012). In a case study on methane-air system, Magina et al. (2019) also showed that the non-premixed flame time lag is about a factor of two smaller than a premixed flame of the same length. In the low Strouhal number regime, the shorter time lag for non-premixed flame, as compared to the premixed flame, was attributed to the fact that the axial distribution of heat release is more uniform for a premixed flame as compared to a non-premixed flame. The mass burning rate (and subsequently the heat release rate) was shown to attain the highest value near burner outlet (or flame base) for a non-premixed flame; thus the spatially integrated heat release is controlled by the near burner outlet region for a non-premixed flame, resulting in a comparatively lower time lag as compared to its premixed counterpart. Premixed flame of constant burning velocity was shown to have a fixed normalized time lag value. To summarize, in the present context, we shall restrict ourselves to the low Strouhal number regime: Any investigation on intrinsic- 
acoustic mode coupling for different parameter domains of Strouhal number (i.e. $S t=\mathrm{O}(1)$ or $S t>1)$ will necessitate the usage of different flame transfer functions, as given in Lieuwen (2012), which calls for a detailed scrutiny in the future.

Apart from two Eqs. $(5,6)$ at the boundaries, we have two jump conditions for the acoustic pressure and flow velocity at the flame location $x=x_{q}$. When the mean flow is neglected, these jump conditions take the following form (Bauerheim et al., 2015),

$$
\begin{gathered}
\tilde{p}_{1}(x)=\tilde{p}_{2}(x), \text { and } \\
S_{1} \tilde{u}_{1}(x)+\left((\gamma-1) / \rho_{1} c_{1}^{2}\right) \tilde{Q}=S_{2} \tilde{u}_{2}(x) .
\end{gathered}
$$

The homogeneous equations (5-8) can be presented in a matrix form (e.g., Mukherjee and Shrira, 2017). For a nontrivial solution of the eigen-value problem to exist, the determinant of the matrix must be zero; this condition yields the dispersion relation for the 1-D resonator, a compact expression of which reads (Mukherjee and Shrira, 2017),

$$
\begin{gathered}
{\left[\left(S_{2} / S_{1}\right)\left(\rho_{1} / \rho_{2}\right)\left(c_{1} / c_{2}\right)+1\right] \cos \left(\left(k_{2}-k_{1}\right) x_{q}-k_{2} L\right)+} \\
{\left[\left(S_{2} / S_{1}\right)\left(\rho_{1} / \rho_{2}\right)\left(c_{1} / c_{2}\right)-1\right] \cos \left(k_{2} L-\left(k_{1}+k_{2}\right) x_{q}\right)+2 n e^{i \omega \tau} \sin k_{1} x_{q} \sin k_{2}\left(x_{q}-L\right) \equiv f(\omega)=0 .}
\end{gathered}
$$

We make use of the dispersion relation (9) for the analytical developments of this paper.

In Mukherjee and Shrira (2017) (Figs. 3 and 4) there was an example, where the first intrinsic mode is strongly decaying in the range of small $n$ and, as $n$ is increased, the first acoustic mode becomes unstable at the frequency of the first intrinsic mode, whereas, the first intrinsic mode remains stable.

Here we consider the same situation as in Fig. 4 by Mukherjee and Shrira (2017), as we gradually increase $n$ and track down the loci of the first intrinsic mode and first acoustic mode on the complex frequency plane. Fig. 2 (a), (b), (c), (d) and (e) present contour plots of $|f(\omega)|$ (based on Eq. (9)) for $\tau=3 \mathrm{~ms}, x_{q}=L / 2$ and neglecting the effects of cross-section and temperature jump across the flame. Panel (a2) of Fig. 2 depicts the strongly decaying intrinsic mode at $n=0.025$. Fig. 2 (b) shows the case when $n$ is increased to 0.25 and the first acoustic mode decays, whereas the decay rate of the first intrinsic mode reduces (compared to an uncoupled intrinsic mode). In Fig. 2 (c), $n$ is increased to 0.5, keeping $\tau=3 \mathrm{~ms}$. The decay rate of the first acoustic mode increases further, whereas, the decay rate of the first intrinsic mode reduces. Thus, these two modes come closer to each other on the complex frequency plane. Another important aspect is the crossover of frequency, that is the frequencies of the first acoustic and first intrinsic modes cross each other and the first acoustic mode attains higher frequency than the first intrinsic mode. Note that in Figs. 2 (b) and (c) the decay rate of the first intrinsic mode is always larger than the decay rate of the first acoustic mode, even if they come very close to each other on the complex frequency plane. In Fig. 2 (d) $n$ is further increased to 0.8 keeping $\tau$ the same. The first acoustic mode becomes marginally unstable at this value of $n$ at the frequency of the first intrinsic mode in the asymptotic limit of small $n$. 
The decay rate of the first intrinsic mode is reduced further, but the mode does not cross the neutral curve. Finally, Fig. 2 (e) generated for $n=1.0$, shows that the acoustic mode becomes unstable, whereas, the intrinsic mode remains decaying.

Fig. 2 illustrates the basic scenario of intrinsic-acoustic mode coupling on the complex frequency plane: with a gradual increase of $n$ the initially decaying acoustic mode becomes unstable at the frequency of the intrinsic mode (taken in the limit of small $n$ ). For convenience we have shown an example in Fig. 2, where we have only one unstable solution. However, there are also such instances where we find two unstable solutions, one for coupled-intrinsic and another for the coupled-acoustic, details of which will be given in $\S 4$.

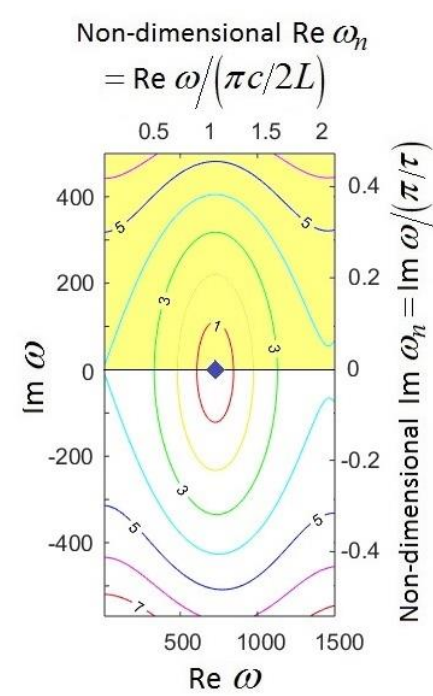

(a1)

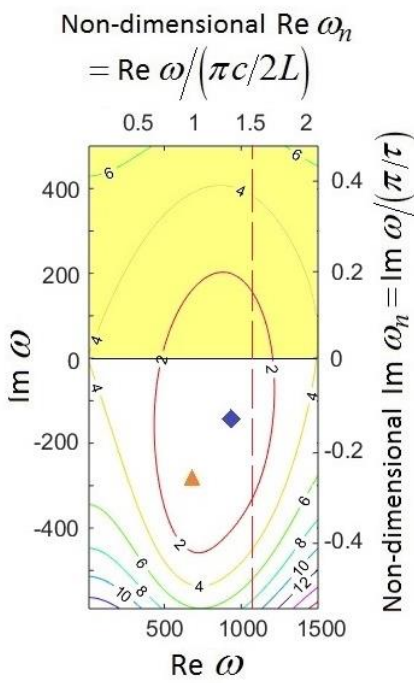

(c)

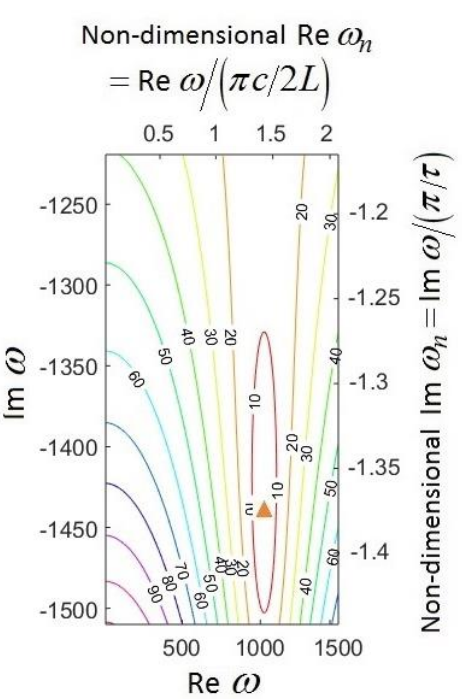

(a2)

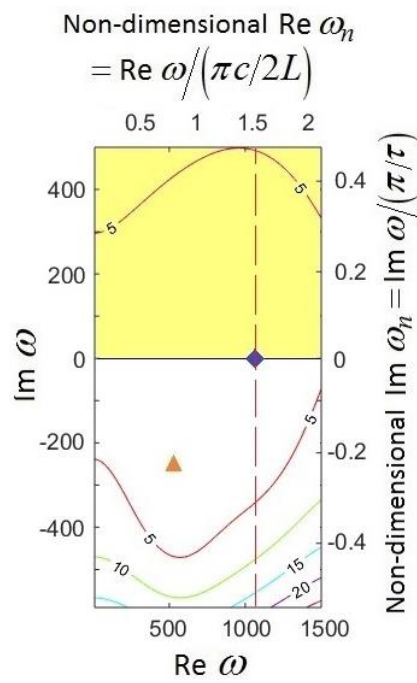

(d)

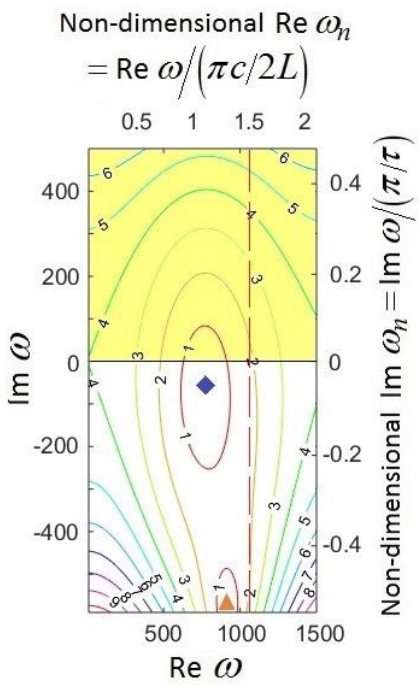

(b)

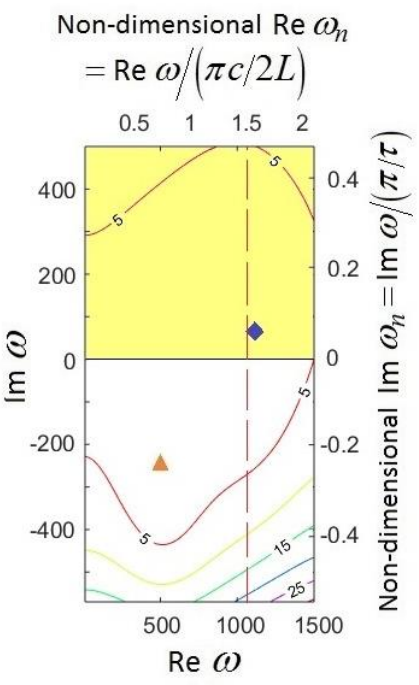

(e)

Figure 2: Contour plot of $|f(\omega)|$ as per Eq. (9) for (a) $n=0.025$, (b) $n=0.25$, (c) $n=0.5$, (d) $n=0.8$, (e) $n=1.0$ at $\tau=3.0 \mathrm{~ms}$, for the particular case $x_{q}=L / 2$. (a1), (a2) both are parts of the panel (a). Because of the large difference in the imaginary parts of the frequency, they are 
shown in two different panels. The parameters used for this plot are (Vishnu et al., 2015): the length $L$ is $0.75 \mathrm{~m}$, the uniform cross-section $S$ is $0.0016 \mathrm{~m}^{2}$, the temperature $T$ is assumed uniform and equal to $297 \mathrm{~K}\left(c_{1}=c_{2}=c=345 \mathrm{~m} / \mathrm{s}\right)$. The domain of instability is lightly shaded (yellow online). Diamonds (blue online) and triangles (orange online) indicate the acoustic and intrinsic modes, respectively. We assume that the mode identities do not change. Dashed vertical lines indicate the intrinsic mode frequency in the limit of small $n$, given by $\omega^{i}=\left(2 m^{i}+1\right)(\pi / \tau)$, where $m^{i}$ is the mode number of the intrinsic mode. This figure provides evidence of intrinsic-acoustic mode coupling.

\section{Pressure profiles of acoustic and intrinsic modes during coupling}

The idea that the modes have fixed identities and it is always possible to find and label them as acoustic or intrinsic is an oversimplification, helpful only in a certain part of the parameter space. Because the coupled acoustic mode, behaves exactly like an intrinsic mode as it approaches the instability boundary, from now on we will refer to both acoustic and intrinsic modes as coupled modes. However, to indicate the "initial" identity of these modes, i.e. identity for small $n$ where the modes are well separated, we use identifiers: coupled mode ('born acoustic' or in short $B A$ ) and coupled mode ('born intrinsic' or in short $B I$ ). An insight, to "reveal" the identity of the modes can be provided by a detailed study of "evolution" of their pressure profiles as the solutions "move" on the $n-\tau$ plane because of varying $n$. Using Eqs. (3-6), the pressure profiles upstream and downstream of the flame can be written as (for uniform temperature and cross-section)

$$
p_{1}(x)=2 B_{1} \cos (k x) \text {, and } p_{2}(x)=-2 i e^{-i k L} B_{2} \sin \{k(x-L)\},
$$

where $B_{2}$ in (11) is related to $B_{1}$ in (10) as, $B_{2}=i e^{i k L}\left[\cos \left(k x_{q}\right) / \sin \left\{k\left(x_{q}-L\right)\right\}\right] B_{1}$ (using (7)). The pressure downstream of the flame, can be restructured as,

$$
p_{2}(x)=2 B_{1}\left[\cos \left(k x_{q}\right) / \sin \left\{k\left(x_{q}-L\right)\right\}\right] \sin \{k(x-L)\} .
$$

Eqs. (10) and (12) together describe the acoustic pressure distribution in the resonator. Thus, we can examine the pressure profiles of the coupled modes. Figs. 3 (a-h) show such pressure profile plots for the same value of $\tau$ ( $\tau=3 \mathrm{~ms}$ ) as shown in the example of $\S 3$. The first intrinsic and first acoustic modes are coupled in this case. The cross-section and temperature are assumed to be uniform here.

In Fig. 3 (a, b) $n$ is extremely small and, thus, the intrinsic mode (BI) is heavily decaying and does not feel the boundaries of the combustor (for more details see Mukherjee and Shrira, 2017), as can be seen from the pressure profile. The coupled mode (BA), in contrast, is determined by the combustor boundaries and has zero phase. As $n$ is increased to 0.1, Fig. 3 (c, d) shows that the coupled mode (BI) becomes less localized and starts feeling the combustor boundaries. However, this mode is still heavily decaying. The decay rate of the coupled mode (BI) reduces and it comes closer to the coupled mode (BA). The coupled mode (BA) keeps on 
to be determined by the combustor boundaries. At $n=0.4$ (Fig. 3 (e, f)) the coupled modes BI and BA come closer to each other and attain almost equal frequencies, and thus, the mode "trajectories" on the complex frequency plane start "crossing" each other. The coupled mode (BI) still maintains higher decay rate than the coupled mode (BA). An apparent dip/hump is visible in the coupled mode (BI/BA) pressure profile. Note that at this range of $n$ the pressure profiles for the coupled modes (BI and BA) look almost identical. This is a manifestation of coupling. The phase angle for the coupled mode (BI) pressure remains higher than that of the coupled mode (BA). As $n$ is increased to 0.8, (see Fig. $3(\mathrm{~g}, \mathrm{~h})$ ), the coupled mode (BA) becomes unstable at the frequency of the coupled mode (BI) in the asymptotic limit of small $n$, whereas, the coupled mode (BI) comes closer to the neutral curve as well. The phase angle for the coupled mode (BA) becomes zero again, whereas, the coupled mode (BI) pressure profile manifests higher phase angle. The difference between pressure profile hump and dip for these two modes remains substantial.

We have thus performed a comparison of the pressure profiles of a coupled pair of an intrinsic and acoustic mode. A coupled mode (BA) decays for small $n$ and then, both the coupled modes (BI and BA) approach the neutral curve with the increase of $n$. In the limit of small $n$ (see Fig. 3 (a)) pressure profiles of the coupled modes (BI and BA) are very different. When the frequencies of the two modes cross on the complex frequency plane, the pressure profiles of coupled modes (BI and BA) appear almost identical. This is our key observation of the pressure profile examination. 


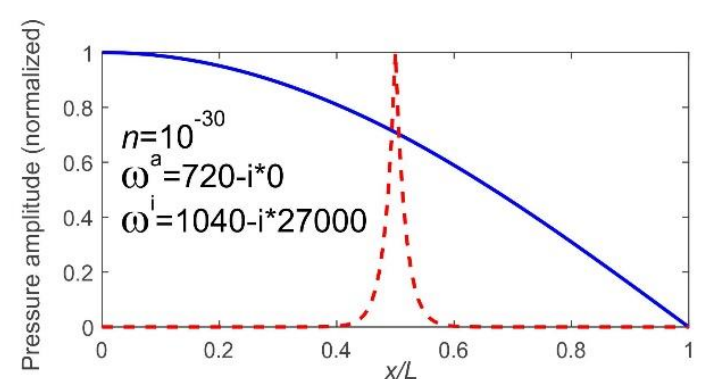

(a)

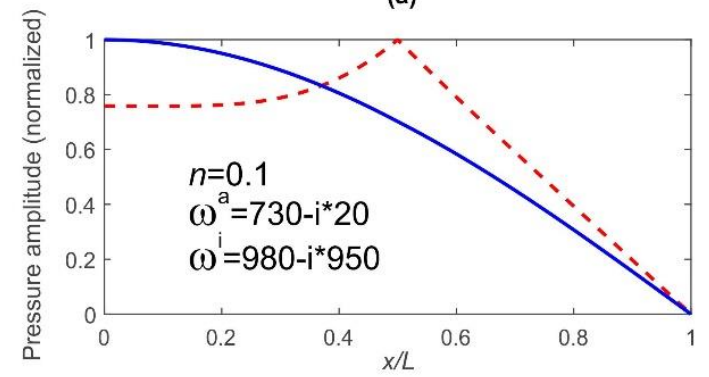

(c)

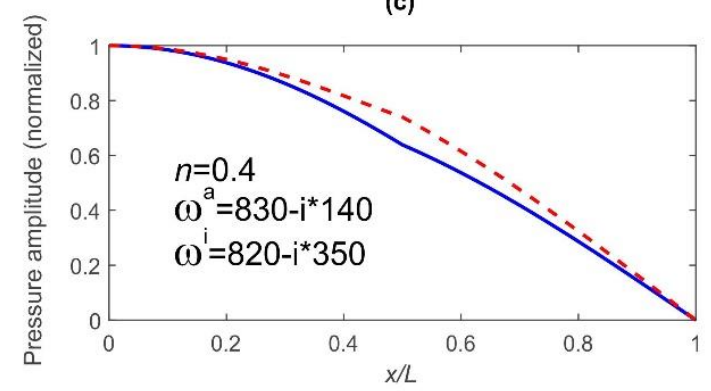

(e)

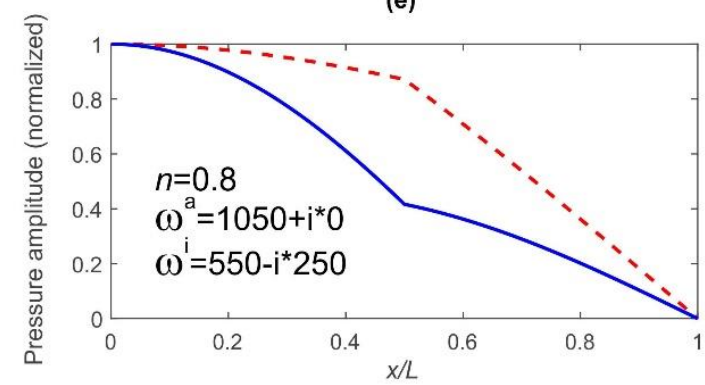

(g)

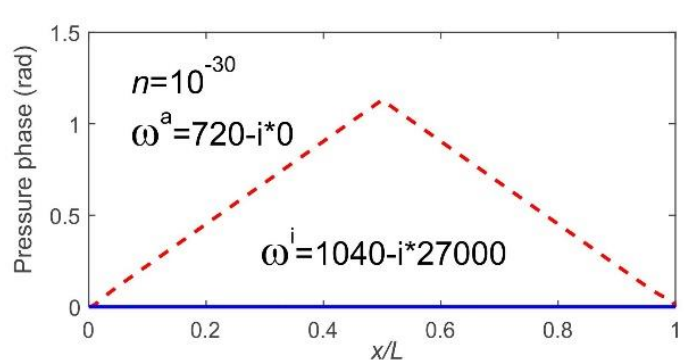

(b)

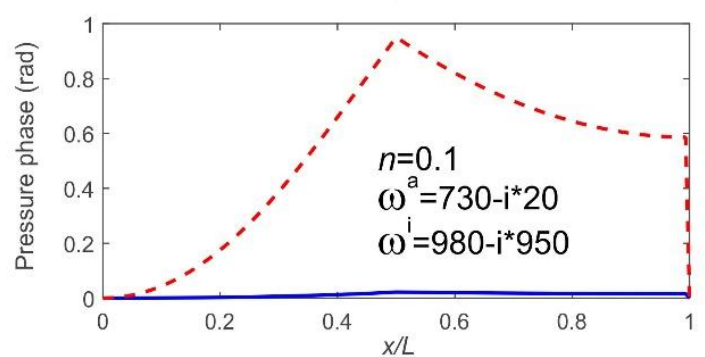

(d)

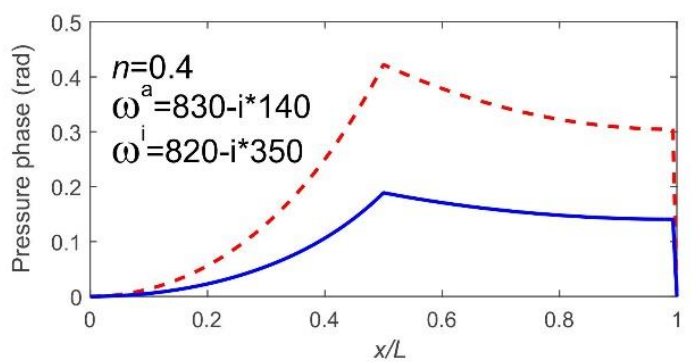

(f)

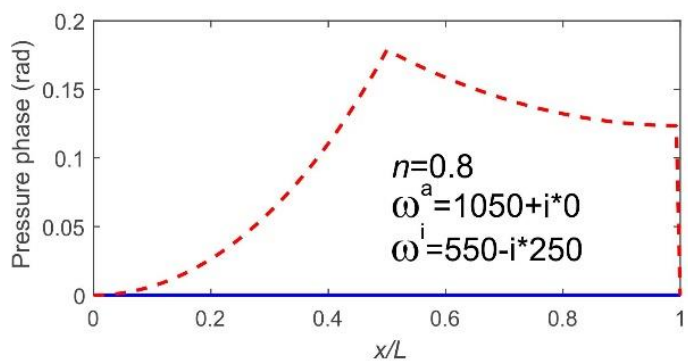

(h)

Figure 3: Normalized pressure amplitude and phase in radians of coupled BI and coupled BA modes based on Eqs. (10) and (12), for $x_{q}=L / 2, \tau=3 \mathrm{~ms}$. The left and right columns of the figure panels represent the pressure amplitude and phase, respectively. The cross-section and temperature are assumed to be uniform. The dashed (red online) and solid (blue online) lines represent the coupled BI mode and coupled BA mode, respectively.

We have seen that comparison of mode shapes could be a reliable option for differentiating the modes when the modes are not coupled (refer appendix A). We then have pure acoustic and pure intrinsic mode instabilities. However, when intrinsic and acoustic modes are potentially coupled and correspondingly, they are closer to each other on the complex frequency plane, their mode shapes become hardly distinguishable, as illustrated in Figs. 3 (a-h). Thus, we can claim that: (i) coupling does manifest itself in the pressure profiles and (ii) differentiation of intrinsic and acoustic coupled modes is questionable and not particularly helpful. The mode identities are clear-cut only outside the domain of strong coupling in the parameter space. 
Possible difficulties in identifying the nature of modes based on their mode shapes were also discussed by Emmert et al. (2016). Note that in $\S 2,3$ examples we showed a case when two modes cross each other on the frequency plane, which may not be true in other types coupling (see $§ 4)$.

\section{Mathematical model of intrinsic-acoustic mode coupling}

In this section, we try to describe intrinsic-acoustic mode coupling employing a simple mathematical model. We will solve the dispersion relation in the vicinity of an acoustic mode, with an intrinsic mode nearby the chosen acoustic mode in the $n-\tau$ space. Thus, we identify the locus of the acoustic and the intrinsic mode that interacts with it in the $n-\tau$ space. We also outline the possible scenarios under which either born acoustic mode alone or both born acoustic and born intrinsic modes become unstable. While in $\S 4.1$, we derive coupling picture for any acoustic mode in general, we also keep in mind that not all acoustic mode couple to the intrinsic mode and some of them manifest pure acoustic instability. Thus, in this section, we refer these modes as acoustic and intrinsic, and from $\S 4.2$ onwards, we use the nomenclature coupled mode BA and BI, to describe coupling.

\subsection{Derivation of the simplest governing dispersion relation for coupling}

It is reasonable to assume that with a small change of parameters the acoustic modes do not deviate far from the neutral curve (in terms of growth/decay rate). Hence, assuming the deviation of the frequency $\Delta \omega$ to be small and using our original dispersion relation (9), we can find the locus of the acoustic mode, as well as, the nearby intrinsic modes. Let $\omega_{0}^{a}$ be the frequency of an acoustic mode of the closed-open combustor in the absence of flame (but with both temperature and cross-section jumps), then the perturbed frequency in the vicinity of the neutral curve can be written as $\omega^{a}=\omega_{0}^{a}+\Delta \omega$. In the present context, we assume the perturbation $\Delta \omega$ to be small compared to $\omega_{0}^{a}$. On substituting the ansatz $\omega^{a}=\omega_{0}^{a}+\Delta \omega$ into the general dispersion relation (9) and expanding it in powers of $\Delta \omega$ and neglecting all the terms higher than order 2 , the dispersion relation can be reduced to a quadratic equation,

$$
A+B(\Delta \omega)+C(\Delta \omega)^{2}=0 .
$$

where, the coefficients, $A, B$ and $C$ in (13) are,

$$
\begin{aligned}
& A=(\alpha+1) \cos \left(\beta_{1} \omega_{0}^{a}\right)+(\alpha-1) \cos \left(\beta_{2} \omega_{0}^{a}\right)+2 n e^{i \omega_{0}^{a} \tau} \sin \left(\frac{\omega_{0}^{a} x_{q}}{c_{1}}\right) \sin \left(\frac{\omega_{0}^{a}\left(x_{q}-L\right)}{c_{2}}\right), \\
& B=-(\alpha+1) \beta_{1} \sin \left(\beta_{1} \omega_{0}^{a}\right)-(\alpha-1) \beta_{2} \sin \left(\beta_{2} \omega_{0}^{a}\right)+
\end{aligned}
$$

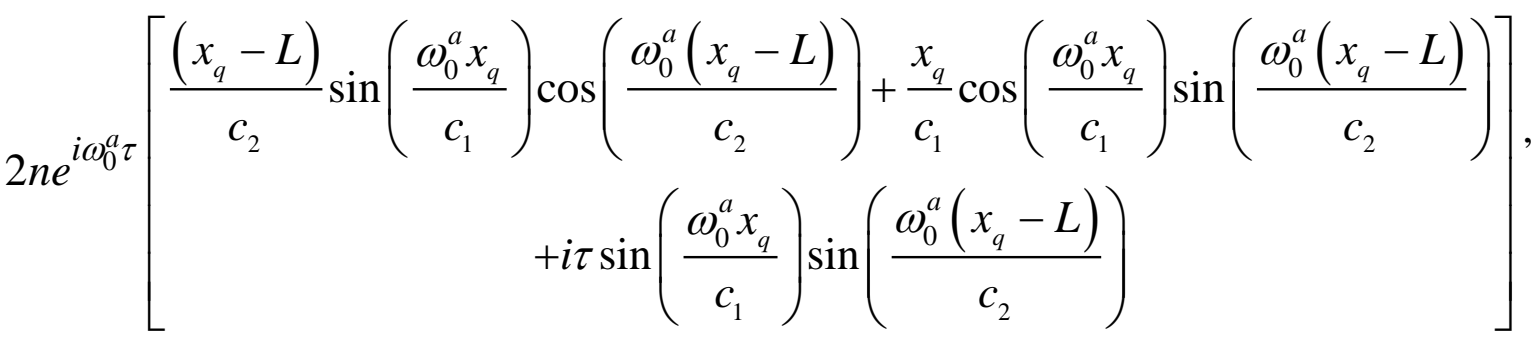




$$
\begin{aligned}
& C=-(\alpha+1) \frac{\beta_{1}^{2}}{2} \cos \left(\beta_{1} \omega_{0}^{a}\right)-(\alpha-1) \frac{\beta_{2}^{2}}{2} \cos \left(\beta_{2} \omega_{0}^{a}\right)+ \\
& 2 n e^{i \omega_{0}^{a} \tau}\left[\begin{array}{c}
\left.-\frac{\left(x_{q}-L\right)^{2}}{2 c_{2}^{2}}-\frac{x_{q}^{2}}{2 c_{1}^{2}}-\frac{\tau^{2}}{2}\right\} \sin \left(\frac{\omega_{0}^{a} x_{q}}{c_{1}}\right) \sin \left(\frac{\omega_{0}^{a}\left(x_{q}-L\right)}{c_{2}}\right)+L \\
c_{1} c_{2} \\
\cos \left(\frac{\omega_{0}^{a} x_{q}}{c_{1}}\right) \cos \left(\frac{\omega_{0}^{a}\left(x_{q}-L\right)}{c_{2}}\right)+\frac{i \tau x_{q}}{c_{1}} \cos \left(\frac{\omega_{0}^{a} x_{q}}{c_{1}}\right) \sin \left(\frac{\omega_{0}^{a}\left(x_{q}-L\right)}{c_{2}}\right) \\
+\frac{i \tau\left(x_{q}-L\right)}{c_{2}} \sin \left(\frac{\omega_{0}^{a} x_{q}}{c_{1}}\right) \cos \left(\frac{\omega_{0}^{a}\left(x_{q}-L\right)}{c_{2}}\right)
\end{array}\right] .
\end{aligned}
$$

Once using (13) the correction to the frequency is obtained, the two frequencies (of acoustic mode and nearby intrinsic mode) can be obtained using the relation: $\omega^{a}=\omega_{0}^{a}+\Delta \omega$. This approach tells us whether in the $n-\tau$ space any of the intrinsic modes is in the vicinity of the acoustic mode and is coupled with it. Further examples show that strong mode coupling occurs for finite $n$ close to the threshold value. For small $n$ acoustic mode decays (for negative Rayleigh index) and does not feel the intrinsic mode. For subcritical and threshold values of $n$ it interacts with the intrinsic mode that comes closer in the $n-\tau$ space and becomes unstable at the same frequency as that of the intrinsic mode, whereas the intrinsic mode either becomes unstable or remains stable for larger $n$. Once unstable or close to being unstable, acoustic mode behaves exactly like the intrinsic mode, it is coupled with.

\subsection{The four types of intrinsic-acoustic mode coupling}

The analytical solution (13) can be plotted on the $n-\tau$ plane and we can perform a visual inspection on whether or not acoustic and intrinsic modes come close enough to one another for the coupling to occur. Such plots can be generated for any chosen acoustic mode with any given value of $\tau$. For a chosen specific $\tau, n$ will be increased in small steps from zero to a finite value and the corresponding acoustic and the nearby intrinsic mode frequencies will be calculated using (13). Finally, we will obtain the loci of the acoustic and intrinsic modes on the complex frequency plane with $n$ treated as a parameter. The analytical solution provided by the quadratic equation (13) will be verified with the numerical solution of Eq. (9). 


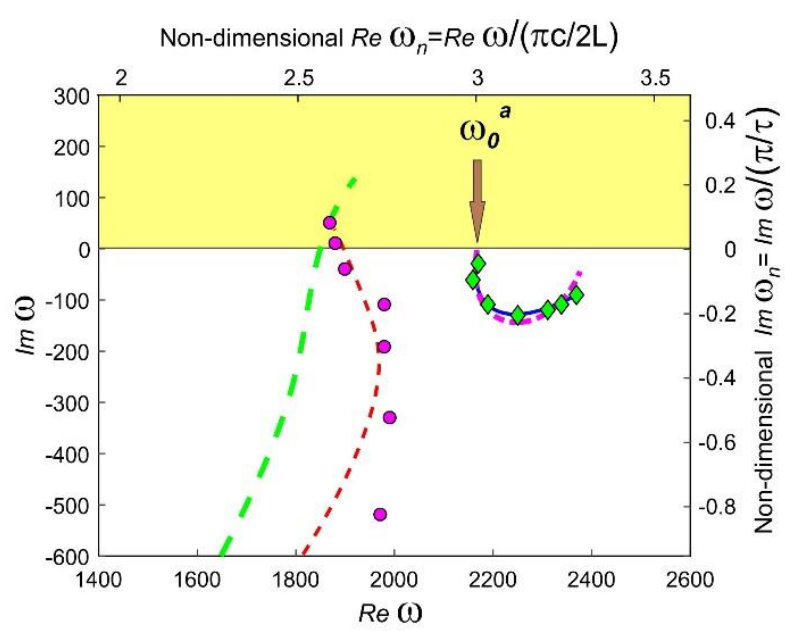

(a)

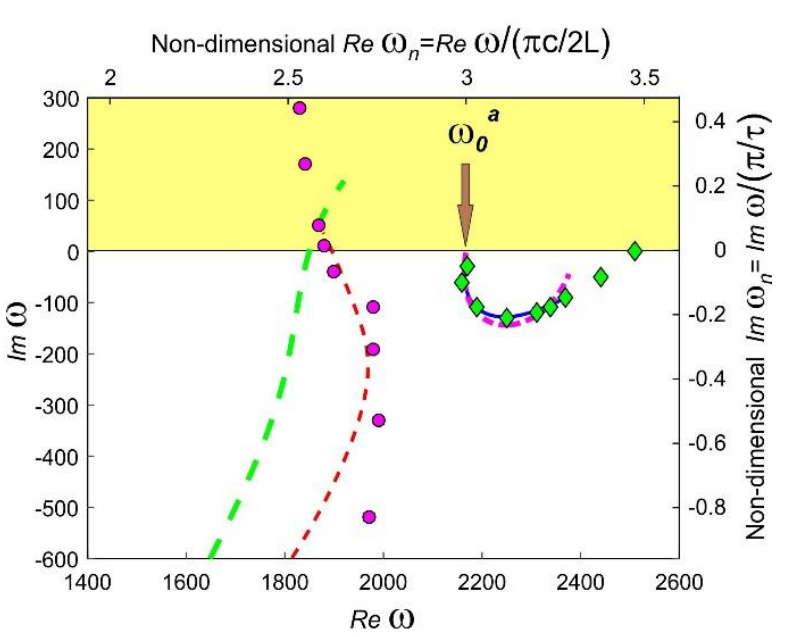

(b)

Figure 4: The first type of coupling: Coupled intrinsic-acoustic modes for $x_{q}=L / 2, \tau=5 \mathrm{~ms}$, $m^{a}=1, m^{i}=1$. Analytical solutions based on the quadratic equation (13) for coupled modes (BA) and (BI) are plotted by dashed-dotted (magenta online) and light, thick dashed (green online) lines, respectively. Analytical solutions based on the quartic equation (14) for coupled modes (BA) and (BI) are shown by continuous (blue online) and darker, thin dashed (red online) lines, respectively. The diamond (green online) and circular (magenta online) dots represent their numerical counterparts obtained by solving Eq. (9). Due to identically good predictions, the solutions of coupled BA modes as per (13) and (14), almost overlap each other. Lightly shaded region (yellow online) is the domain of instability. The arrow indicates the frequency of acoustic mode $\left(\omega_{0}^{a}\right)$ of the closed-open combustor in the absence

of flame. For analytical solutions $n$ is varied from 0.0 to 1.0 for both (a) and (b). For numerical solutions $n$ is varied from 0.0 to 1.0 in (a) and from 0.0 to 4.0 in (b). The crosssection and temperature are assumed uniform. Other parameters are the same as in Fig. 2.

Fig. 4 shows an example of such a plot for the second acoustic mode ( $\left.m^{a}=1\right)$, when $\tau=5 \mathrm{~ms}$ and flame is at $x_{q}=L / 2$. The figure illustrates coupling between the second acoustic and the second intrinsic mode $\left(m^{i}=1\right)$. In this particular case, the mode numbers for the acoustic and intrinsic modes involved in coupling are the same. Figure makes it obvious that as $n$ increases from 0 to 1.0, the coupled mode (BA) decays initially, as can be seen from the "mode trajectory" on the complex frequency plane and becomes unstable at a frequency predicted by $\omega_{c}^{i} \tau=\pi$, whereas, the coupled mode (BI) becomes unstable at the frequency predicted for it in the asymptotic limit of small $n$ (i.e. at a frequency corresponding to $\omega_{c}^{i} \tau=0$ ). Indeed, the coupled mode (BI) becomes unstable at a much lower threshold of $n$ than the coupled mode (BA), We name this form of coupling as the "first type of coupling", where coupled mode (BI) attains instability at a frequency corresponding to $\omega_{c}^{i} \tau=0$ and the coupled mode (BA) becomes unstable at a frequency corresponding to $\omega_{c}^{i} \tau=\pi$ (or $\omega_{c}^{i} \tau=-\pi$ ). Fig. 4 shows a 
good correspondence between analytical (13) and numerical (9) results for the coupled mode (BA), whereas, for the coupled mode (BI), the predictions are not that accurate outside a vicinity of the stability boundary. The analytical solution, however, captures the instability frequency of the coupled mode (BI) on the complex frequency plane accurately. For $n$ higher than 1.0, analytical solution deviates drastically from numerical solution, because the other solution for coupled mode (BI) grows strongly, indicating advent of nonlinearity in the system. This trend is captured in Fig. 4 (b). In this work we are speaking about the nonlinearity in terms of expansion in $\Delta \omega$. Thus, the quadratic analytical model (13) tends to predict a slightly lower instability frequency for the coupled mode (BA), as is evident from Fig. 4 (b). This is a general limitation of the quadratic analytical model (13). For too large $n$ the accuracy deteriorates. However, for the range of $n$ for which linear analysis remains valid, the prediction accuracy is reasonably good.

Another type of coupling is illustrated in Fig. 5, showing the second acoustic mode $\left(m^{a}=1\right)$, when $\tau=4.8 \mathrm{~ms}$ and flame is at $x_{q}=L / 2$. As we increase $n$ from 0 to 1.0 the coupled mode (BA) becomes unstable at $\omega_{c}^{i} \tau=0$, whereas, the coupled mode (BI) tends to become unstable at a higher frequency corresponding to $\omega_{c}^{i} \tau=\pi$. We name this scenario as the "second type of coupling". The $n$-threshold for instability is higher for the coupled mode (BI) than for the coupled mode (BA). Similar to Fig. 4 the quadratic analytical model (13) tracks accurately the trajectory of the numerical solution and instability frequency for the coupled mode (BA). However, for the coupled mode (BI) Fig. 5 (b) shows that the coupled mode (BI) becomes unstable at too high $n$, indicating a possible advent of nonlinearity into the system due to strongly growing coupled mode (BA). This reduces the accuracy of the quadratic analytical model (13) for the coupled mode (BI). Figs. 4 and 5 illustrate that a slight shift in $\tau$ (from 5 to $4.8 \mathrm{~ms}$ ) changes the mode coupling type from the first to the second, which forces either: (i) the coupled BI mode to appear on the neutral segment $\omega_{c}^{i} \tau=0$ and the coupled BA mode to appear on the neutral segment $\omega_{c}^{i} \tau=\pi$ (or $\omega_{c}^{i} \tau=-\pi$ ) or, (ii) the coupled BA mode to appear on the neutral segment $\omega_{c}^{i} \tau=0$ and the coupled BI mode to appear on the neutral segment $\omega_{c}^{i} \tau=\pi$ (or $\omega_{c}^{i} \tau=-\pi$ ). This phenomenon can be seen in Mukherjee and Shrira (2017) (Fig. 12) and will be elaborated in $\S 5$. 


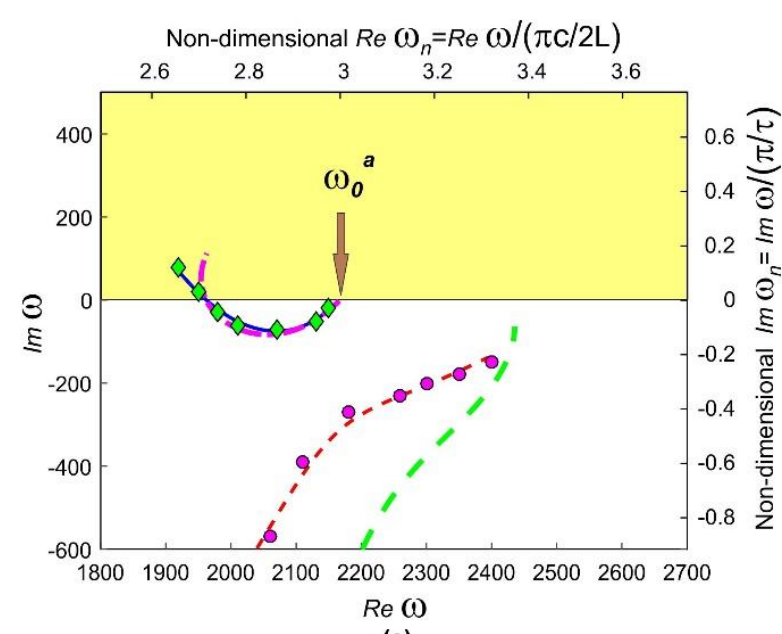

(a)

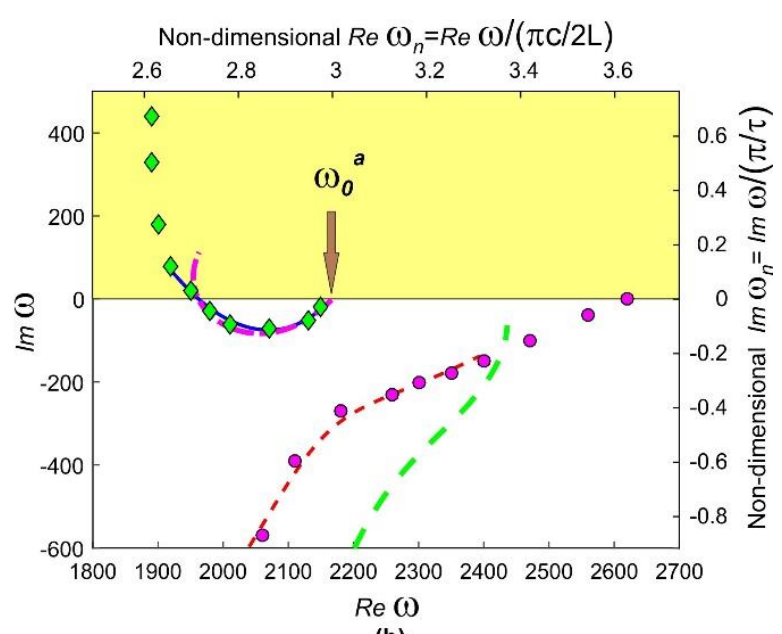

(b)

Figure 5: The second type of coupling: Coupled intrinsic-acoustic modes for $x_{q}=L / 2, \tau$ $=4.8 \mathrm{~ms}, m^{a}=1, m^{i}=1$. For numerical solutions as per (9), $n$ is varied (a) from 0.0 to 1.0 and (b) 0.0 to 10.0. For analytical solutions given by $(13,14), n$ is varied from 0.0 to 1.0 for both (a) and (b). Other parameters and notations are the same as in Fig. 4.

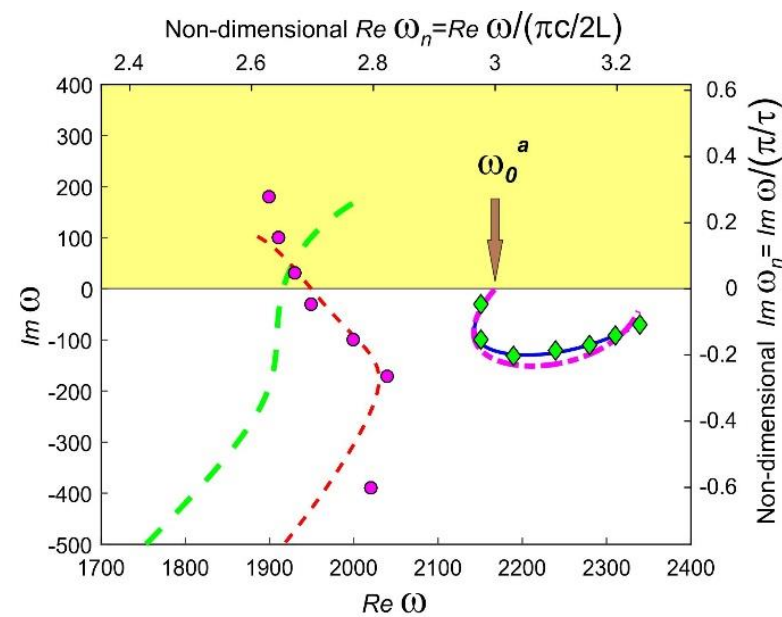

(a)

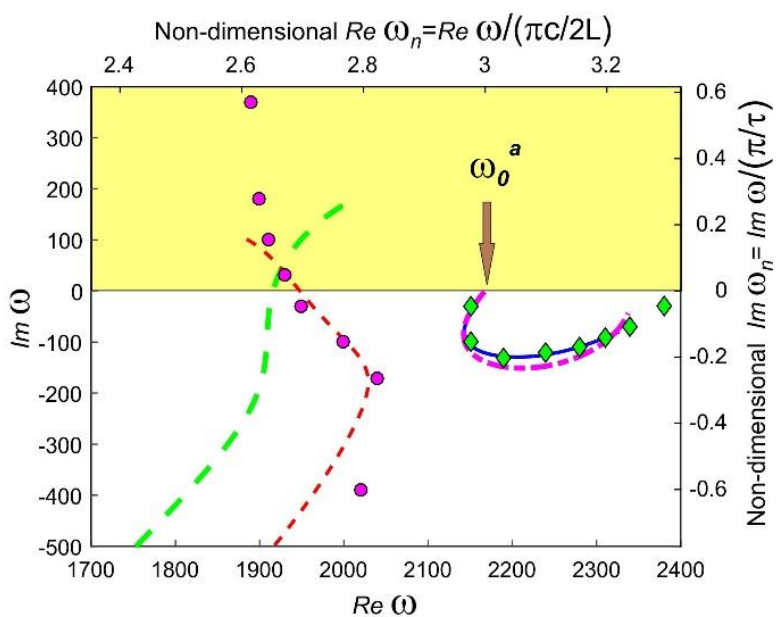

(b)

Figure 6: The third type of coupling: Coupled intrinsic-acoustic modes for $x_{q}=0.3 \mathrm{~m}, \tau$

$=4.85 \mathrm{~ms}, m^{a}=1, m^{i}=1$. For numerical solutions, as per (9), $n$ is varied from (a) 0.0 to 2.5

(b) 0.0 to 7.5 . For analytical solutions given by $(13,14), n$ is varied from 0.0 to 2.0 for both (a) and (b). Other parameters and notations are the same as in Fig. 4. 


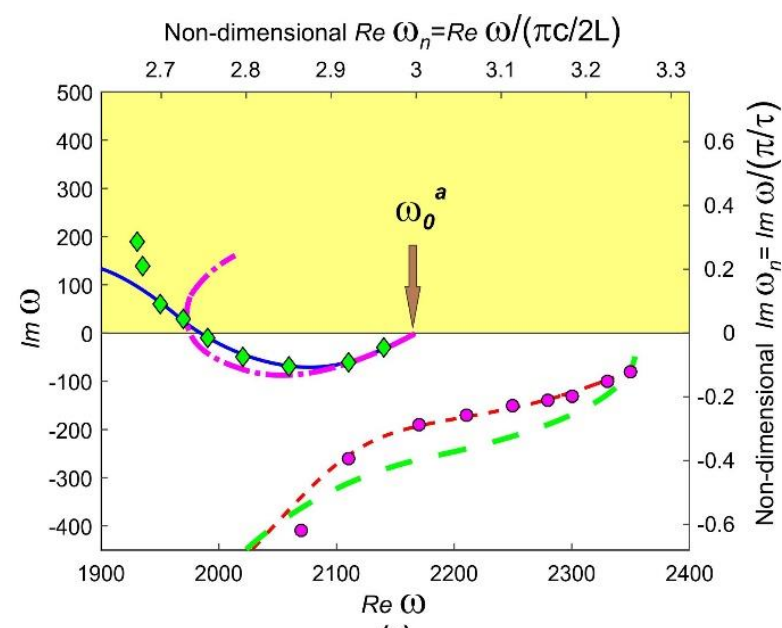

(a)

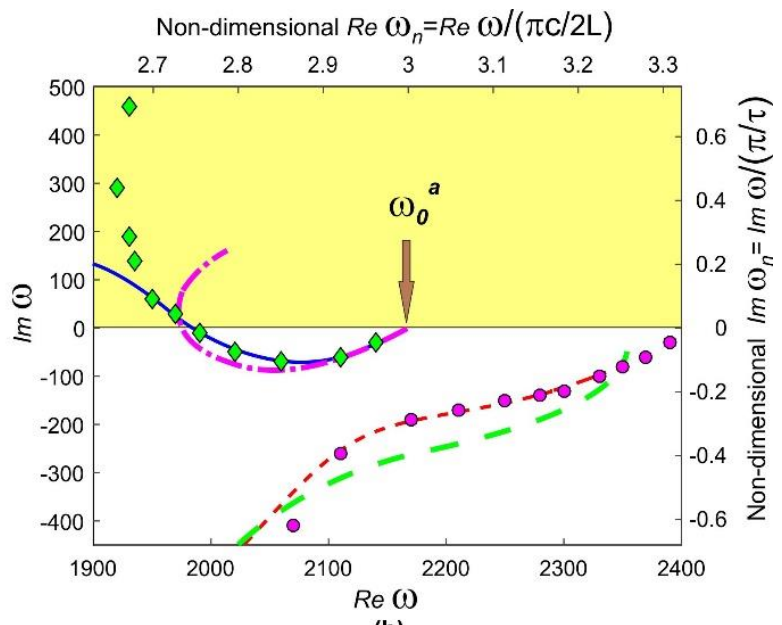

(b)

Figure 7: The fourth type of coupling: Coupled intrinsic-acoustic modes for $x_{q}=0.3 \mathrm{~m}, \tau$

$=4.75 \mathrm{~ms}, m^{a}=1, m^{i}=1$. For numerical solutions as per (9), $n$ is varied from (a) 0.0 to 2.5

(b) 0.0 to 12.5 . For analytical solutions $(13,14), n$ is varied from 0.0 to 2.0 for both (a) and (b). Other parameters and notations are the same as in Fig. 4.

There are two more types of possible coupling, as illustrated in Figs. 6 and 7. Both figures are generated for the second acoustic mode $\left(m^{a}=1\right)$, when the flame is at $x_{q}=0.3 \mathrm{~m}$. Fig. 6 is for $\tau=4.85 \mathrm{~ms}$, whereas, Fig. 7 is for $\tau=4.75 \mathrm{~ms}$. It can be seen from Fig. 6 that as $n$ is varied from 0 to 2.0, the coupled mode (BI) becomes unstable at intrinsic mode frequency $\omega_{c}^{i} \tau=0$, whereas, the coupled mode (BA) tends to remain asymptotically stable at a frequency corresponding to $\sin \left(k_{1} x_{q}\right)=0$ as per Eq. (1). We interpret this as the "third type of coupling", where in spite of mode coupling we find only one unstable mode, whereas, the other mode remains asymptotically stable at a frequency corresponding to $\sin \left(k_{1} x_{q}\right)=0$ or $\sin \left(k_{2}\left(x_{q}-L\right)\right)=0$ as per Eq. (1). Eq. (1) suggests that even though we considered the primary solution $\sin \left(\omega_{c}^{i} \tau\right)=0$ to specify our neutral curve, we have two more solutions corresponding to $\sin \left(k_{1} x_{q}\right)=0$ or $\sin \left(k_{2}\left(x_{q}-L\right)\right)=0$, both of which correspond to $n_{t h} \rightarrow \infty$, as per (2) and thus they do not manifest on the neutral curve. When this form of coupling occurs, it is easy to misinterpret the instability as a pure intrinsic mode instability and wrongly assume that there is no coupling. A simple test to distinguish between uncoupled domain (manifesting pure intrinsic instability) and third or fourth types of coupled domain will be suggested here. If the range of $n_{t h}$ values for instability in the domain is found to be very close to zero, we interpret it as a coupled domain. Because of strong decay of intrinsic modes, they cannot be unstable at such small $n_{t h}$ for any practical range of $\tau$. If $n_{t h}$ within the domain is on the higher side, say, $(\mathrm{O}(1))$, then we interpret it as an uncoupled domain with pure intrinsic instability. Fig. 6 shows that analytical model (13) provides a good prediction for instability 
frequency of the coupled mode (BI), even though it fails to track the locus of the mode on complex frequency plane with a good accuracy. The locus of coupled mode (BA) is captured accurately by the quadratic model (13). Fig. 6 (b) shows that this mode remains stable for $n$ $=7.5$, at the frequency specified by $\sin \left(k_{1} x_{q}\right)=0$, as per Eq. (1). Further increase of $n$ (not shown here) does not allow this mode to become unstable.

In Fig. 7, an increase of $n$ from 0 to 2.0 makes the coupled mode (BA) unstable at the intrinsic mode frequency $\omega_{c}^{i} \tau=0$, whereas, the coupled mode (BI) tends to remain asymptotically stable at a frequency corresponding to $\sin \left(k_{1} x_{q}\right)=0$ as per Eq. (1). We refer to this scenario as the "fourth type of coupling". A closer observation of Fig. 7 shows that the quadratic analytical model (13) tracks the loci of both coupled modes, (BA) and (BI), with a good accuracy. The instability frequency for the coupled mode (BA) is predicted accurately. The other solution for coupled mode (BI) remains asymptotically stable for large $n$, as can be seen from Fig. 7 (b), and hence, we obtain only one unstable solution for this case. Another important aspect of Figs. 6 and 7 is that as we reduce $\tau$ from $4.85 \mathrm{~ms}$ to $4.75 \mathrm{~ms}$, the type of coupling changes from the third to fourth. Note that the mode numbers for the acoustic and intrinsic modes can be either the same or different for all these scenarios.

Thus, we have derived a compact and 'easy to deal with' quadratic equation model to describe the solutions for the coupled intrinsic-acoustic modes. The four types of coupling have been identified, based on the trajectories of the coupled modes on the complex frequency plane. We can also conclude that the prediction accuracy of our analytical model (13) is quite good in tracking down the locus of the coupled mode (BA) on complex frequency plane. However, because the coupled mode (BI) for small values of $n$ is far away from the neutral curve, the small $\Delta \omega$ assumption in (13) is not valid for the coupled mode (BI) in the range of small $n$. Hence, the loci of the coupled mode (BI) on the complex frequency plane at a significant distance from the neutral curve are often not captured with a good accuracy.

\subsection{Inclusion of higher order terms into the analytical model}

So far we have dealt with the quadratic Eq. (13) to describe the intrinsic-acoustic mode coupling. Even though the accuracy of prediction of our analytical model is good enough for the coupled BA modes, it is hardly so for the coupled BI modes. When intrinsic modes are far from the acoustic modes and also from the boundary of stability on the $n-\tau$ plane, this makes it difficult for the quadratic Eq. (13) to predict its loci accurately on the $n-\tau$ plane. Hence, to improve the analytical model, we derive another equation by taking into consideration next order terms in $\Delta \omega$. Substituting the ansatz $\omega^{a}=\omega_{0}^{a}+\Delta \omega$ in (9) and using the sine and cosine expansions, while retaining terms up to order four in $\Delta \omega$, we arrive at a quartic equation to describe mode coupling similar to the quadratic Eq. (13),

$$
A+B(\Delta \omega)+C(\Delta \omega)^{2}+D(\Delta \omega)^{3}+E(\Delta \omega)^{4}=0 .
$$

The coefficients $A, B, C$ are the same as in Eq. (13); two extra coefficients $D$ and $E$ are: 


$$
\begin{aligned}
& D=(\alpha+1) \frac{\beta_{1}^{3}}{6} \sin \left(\beta_{1} \omega_{0}^{a}\right)+(\alpha-1) \frac{\beta_{2}^{3}}{6} \sin \left(\beta_{2} \omega_{0}^{a}\right)+
\end{aligned}
$$

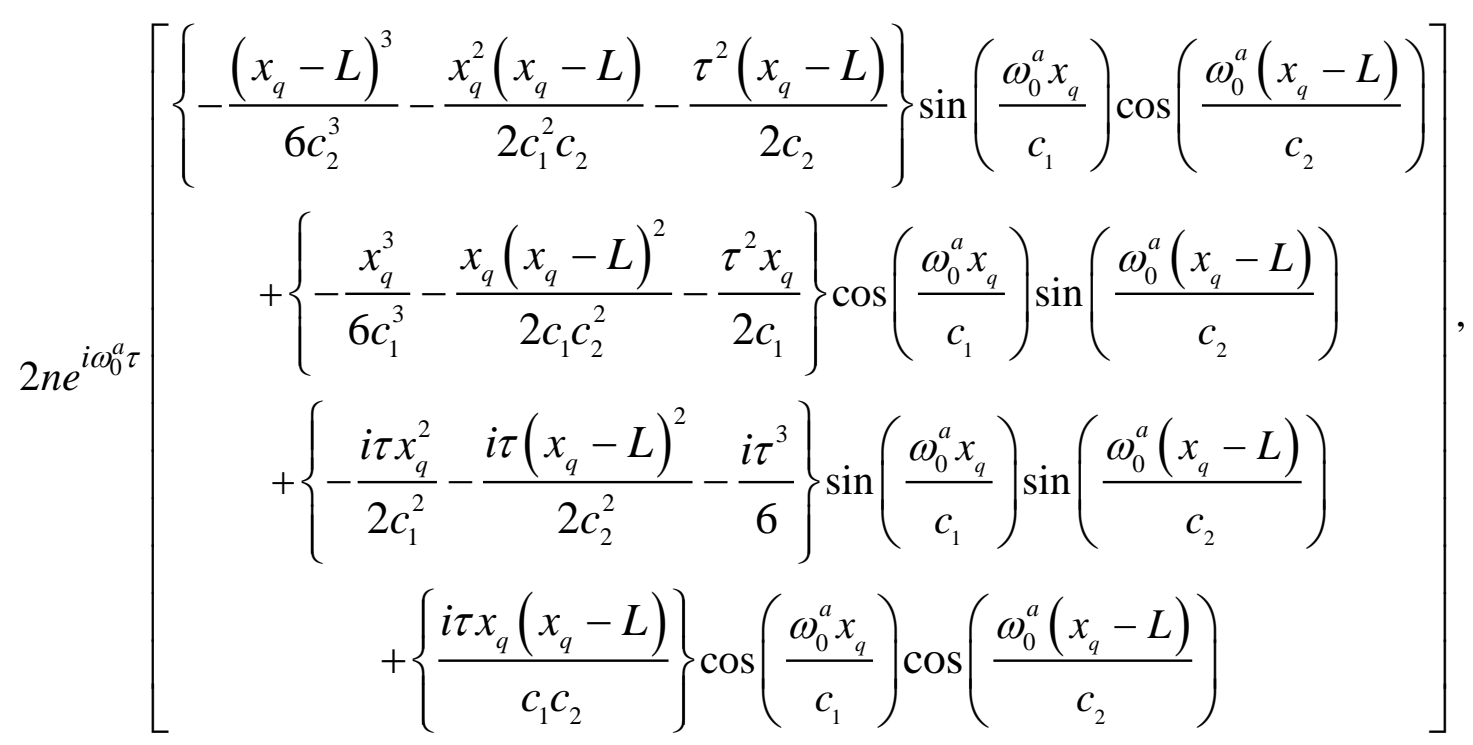

$$
\begin{aligned}
& E=(\alpha+1) \frac{\beta_{1}^{4}}{24} \cos \left(\beta_{1} \omega_{0}^{a}\right)+(\alpha-1) \frac{\beta_{2}^{4}}{24} \cos \left(\beta_{2} \omega_{0}^{a}\right)+ \\
& 2 n e^{i \omega_{0}^{a} \tau}\left[\begin{array}{c}
\left\{\frac{\left(x_{q}-L\right)^{4}}{24 c_{2}^{4}}+\frac{x_{q}^{2}\left(x_{q}-L\right)^{2}}{4 c_{1}^{2} c_{2}^{2}}+\frac{x_{q}^{4}}{24 c_{1}^{4}}+\frac{\tau^{2}\left(x_{q}-L\right)^{2}}{4 c_{2}^{2}}+\frac{\tau^{2} x_{q}^{2}}{4 c_{1}^{2}}+\frac{\tau^{4}}{24}\right\} \sin \left(\frac{\omega_{0}^{a} x_{q}}{c_{1}}\right) \\
\sin \left(\frac{\omega_{0}^{a}\left(x_{q}-L\right)}{c_{2}}\right)+\left\{-\frac{x_{q}\left(x_{q}-L\right)^{3}}{6 c_{1} c_{2}^{3}}-\frac{x_{q}^{3}\left(x_{q}-L\right)}{6 c_{1}^{3} c_{2}}-\frac{\tau^{2} x_{q}\left(x_{q}-L\right)}{2 c_{1} c_{2}}\right\} \cos \left(\frac{\omega_{0}^{a} x_{q}}{c_{1}}\right) \\
\cos \left(\frac{\omega_{0}^{a}\left(x_{q}-L\right)}{c_{2}}\right)+\left\{-\frac{i \tau x_{q}^{3}}{6 c_{1}^{3}}-\frac{i \tau x_{q}\left(x_{q}-L\right)^{2}}{2 c_{1} c_{2}^{2}}-\frac{i \tau^{3} x_{q}}{6 c_{1}}\right\} \cos \left(\frac{\omega_{0}^{a} x_{q}}{c_{1}}\right) \sin \left(\frac{\omega_{0}^{a}\left(x_{q}-L\right)}{c_{2}}\right) \\
+\left\{-\frac{i \tau\left(x_{q}-L\right)^{3}}{6 c_{2}^{3}}-\frac{i \tau x_{q}^{2}\left(x_{q}-L\right)}{2 c_{1}^{2} c_{2}}-\frac{i \tau^{3}\left(x_{q}-L\right)}{6 c_{2}}\right\} \sin \left(\frac{\omega_{0}^{a} x_{q}}{c_{1}}\right) \cos \left(\frac{\omega_{0}^{a}\left(x_{q}-L\right)}{\left.c_{2}\right)}\right) .
\end{array}\right.
\end{aligned}
$$

Thus Eq. (14) can be solved to provide four solutions; one for the BA coupled mode and another for the BI coupled mode in close vicinity. Although Eq. (14) gives rise to four solutions, only two of them have physical significance. We re-consider the same situations as in the plots of Figs. 4-7, but this time using the analytical solution of Eq. (14). Figs. 4-7 demonstrate a very good correspondence between the analytical and numerical solutions, both for the coupled modes (BA) and (BI) (unlike for the quadratic model). The analytical solution for the coupled modes (BI) closely follows the locus predicted by numerical solution, even for the cases where coupled modes (BI) stay far away from the neutral curve. Thus, inclusion of the next order terms into our analytical model significantly improves the prediction accuracy of the loci of the coupled modes on the complex frequency plane. 


\section{Domain of intrinsic-acoustic mode coupling on the $n-\tau$ plane}

Typical intrinsic mode stability maps on the $n-\tau$ plane were discussed in Mukherjee and Shrira (2017). In this section, we identify the domains of intrinsic-acoustic mode coupling on the $n-\tau$ plane. In the domain where the coupling exists, two solutions are dragged close to each other, and, hence, on the $n-\tau$ plane we find two co-existing solutions instead of one.

It is straightforward to identify the domain on the $n-\tau$ plane where two solutions co-exist, as illustrated by Fig. 8 for the second intrinsic mode. The domain bounded by a thick (purple online) vertical line is the domain of coupling, where two solutions co-exist for a particular value of $\tau$. These bounded regions can be seen, for example, near the first and second points of intersections of the neutral curve segments for the main neutral loop. In all other domains outside this coupling domain, there is either no solution (that is, the intrinsic mode under consideration is linearly stable) or only one solution (that is, the intrinsic mode itself is linearly unstable). Another important aspect is that near the intersection points of neutral curve segments, one of the coupled modes (either BI or BA) becomes unstable at smaller (sometimes, at very small) values of $n$, whereas, the other mode becomes unstable at higher (sometimes, very high) values of $n$. Note, that for most practical systems, the solutions lying on $\omega_{c}^{i} \tau=\pi$ and $\omega_{c}^{i} \tau=-\pi$ segments of the neutral curve well above the points of intersection with $\omega_{c}^{i} \tau=0$, may not be feasible, because too high values of $n$ correspond to regimes for which the $n-\tau$ model itself might be invalid. However, any solution lying at or below the point of the segment intersections will be of major interest and of practical significance. It can be seen that near the points of intersection of neutral curve segments, the coupled modes (BA and BI) exhibit an intricate pattern of instability. A slight shift of the time lag might change the type of coupling. In Fig. 8 all four types of coupling are visible. Near the intersection of neutral curve segments, the first and second types of coupling can transmute into third and fourth types of coupling, as is also evident from Fig. 8. It can further be shown that the neutral curve loops on the left-hand side of stability plots (corresponding to smaller values of $\tau$ ), manifest strong coupling, mainly due to small widths of these neutral loops and the presence of closely spaced, almost vertical neutral curve segments $\omega_{c}^{i} \tau=\pi$ and $\omega_{c}^{i} \tau=-\pi$ (Mukherjee, 2017). 


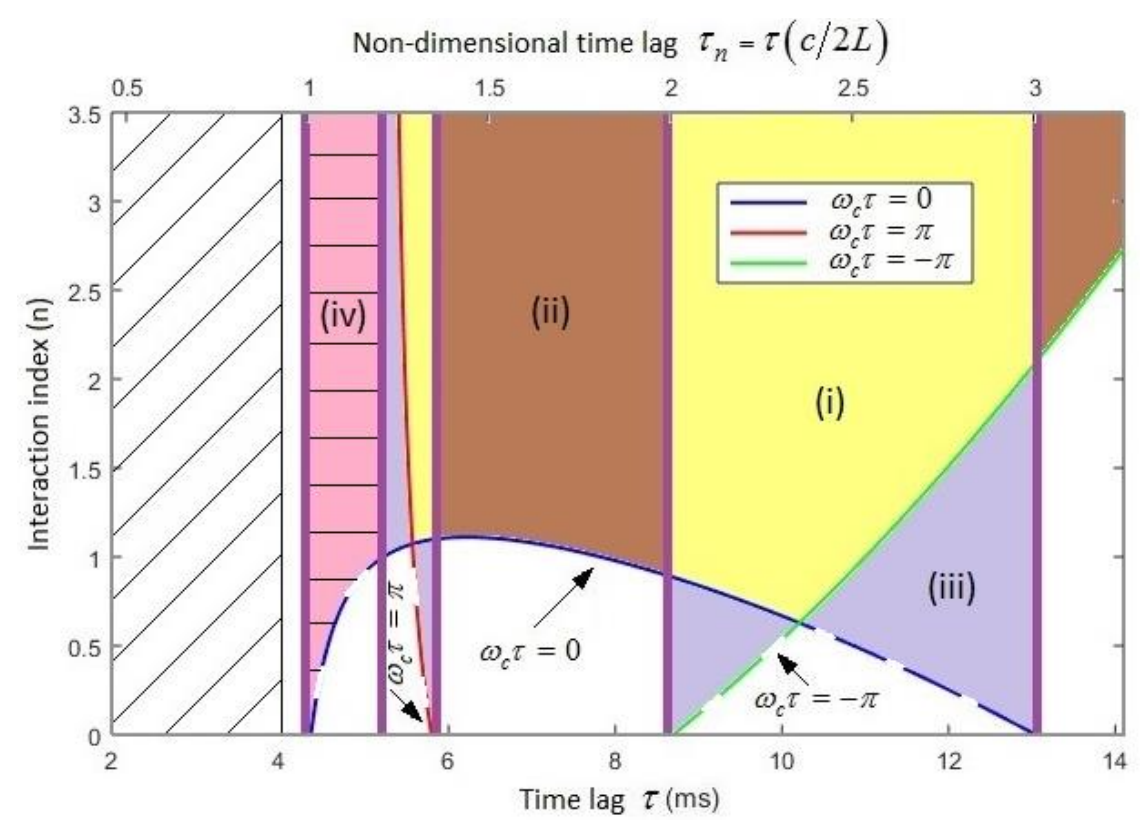

Figure 8: Neutral curve (2) and stability domain on the $n-\tau$ plane for the second intrinsic mode $\left(m^{i}=1\right)$ for $x_{q}=0.3 \mathrm{~m}$ and uniform temperature and cross-section. This figure focuses on the extreme right neutral loop only. The neutral curve segments $\omega_{c}^{i} \tau=0, \omega_{c}^{i} \tau=\pi$ and $\omega_{c}^{i} \tau=-\pi$ are indicated by arrows and plotted by solid lines (blue, red and green, respectively, online). The dashed lines show their continuations which are also exact solutions of (2); we

interpret them as neutral curves for coupled BA modes. The instability domains for uncoupled intrinsic mode and coupled BA modes are marked in a darker shade (ii) (brown online) and medium density shade (iii) (lavender online), respectively. The domain bounded by thick purple vertical lines near the point of intersection of the neutral curve segments represents the domain of intrinsic-acoustic mode coupling. Lightly shaded region (i) (yellow online) is the instability domain of the first and second types of coupling. Shaded, horizontally hatched region (iv) (pink online) marks the instability domain of the third and fourth types of coupling. Hatched area indicates the domain with multiple instability islands narrowing with the decrease of $\tau$. Other parameters are the same as in Fig. 2.

We conclude, that it is possible to identify the domain of coupling solely by visually inspecting the neutral curves of any intrinsic mode. Within the domain of coupling, for a particular value of $\tau$ there are always two solutions: a coupled (BI) and a coupled (BA) (except for the situation with the third and fourth type of coupling, where one solution remains linearly stable). All four types of coupling can be visible on the neutral curve, near the point of intersection, due to a slight change in $\tau$.

\section{Concluding remarks}

Here we have carried out a comprehensive analysis of intrinsic-acoustic mode coupling within the framework of a standard 1-D combustor with $n-\tau$ model of flame heat release. We have identified a new type of instability not reported so far in the literature to the best of our 
knowledge. This novel instability is neither pure acoustic, nor pure intrinsic, rather a product of the coupling between the conventional acoustic and intrinsic modes. To describe it we came up with a quadratic equation model that provides the basis of our analysis of mode coupling. The roots of the quadratic equation yield the frequency and decay/growth rate for the coupled modes. We have tracked the loci of these roots on the $n-\tau$ plane and identified their qualitative behavior. The key observations can be summarized as follows:

i. The idea that the eigen-modes in combustors have fixed immutable identities of either acoustic or intrinsic nature is an oversimplification valid only for small $n$. When at small $n$ the frequencies of an acoustic and nearby intrinsic mode are close, then for moderate values of $n$ mode coupling might occur. When it does occur, the mode behavior becomes incompatible with the idea of fixed identities. The pressure profile of a coupled acoustic mode with an increase of $n$ morphs into the profile typical of intrinsic modes, thus, the coupled mode "origin" becomes irrelevant. The intrinsic-acoustic mode coupling is possible when acoustic pressure and flame heat release are out of phase with each other: then the classical combustion instability is prohibited. We identified four different scenarios of intrinsic-acoustic mode coupling. In all these scenarios, the acoustic mode, due to an increase of $n$ gets coupled to the nearby intrinsic mode and stops behaving like an acoustic mode anymore, by attaining all the features of an intrinsic mode. The coupled mode, which was intrinsic at small $n$ retains all the properties of an intrinsic mode. Hence, we refer the coupled initially intrinsic and coupled initially acoustic modes just as coupled modes; to distinguish them based on their origin, we named them 'born acoustic' (BA) and 'born intrinsic' (BI). Table 1 summarizes the four possible types of mode coupling.

Table 1

\begin{tabular}{|c|c|}
\hline $\begin{array}{l}\text { Type of } \\
\text { coupling }\end{array}$ & Scenario \\
\hline First & $\begin{array}{c}\text { The coupled mode (BI) becomes unstable at a frequency predicted by } \\
\omega_{c}^{i} \tau=0 \text {. The coupled mode (BA) becomes unstable at a frequency } \\
\text { predicted by either } \omega_{c}^{i} \tau=\pi \text { or } \omega_{c}^{i} \tau=-\pi .\end{array}$ \\
\hline Second & $\begin{array}{c}\text { The coupled mode (BA) becomes unstable at a frequency predicted } \\
\text { by } \omega_{c}^{i} \tau=0 \text {. The coupled mode (BI) becomes unstable at a frequency } \\
\text { predicted by either } \omega_{c}^{i} \tau=\pi \text { or } \omega_{c}^{i} \tau=-\pi .\end{array}$ \\
\hline Third & $\begin{array}{l}\text { The coupled mode (BI) becomes unstable at } \omega_{c}^{i} \tau=0 \text {. The coupled } \\
\text { mode (BA) remains asymptotically stable at a frequency } \\
\text { corresponding to } \sin \left(k_{1} x_{q}\right)=0 \text { or } \sin \left(k_{1}\left(x_{q}-L\right)\right)=0 \text { as per Eq. } \\
\text { (1). }\end{array}$ \\
\hline Fourth & $\begin{array}{l}\text { The coupled mode (BA) becomes unstable at } \omega_{c}^{i} \tau=0 \text {. The coupled } \\
\text { mode (BI) remains asymptotically stable at a frequency } \\
\text { corresponding to } \sin \left(k_{1} x_{q}\right)=0 \text { or } \sin \left(k_{1}\left(x_{q}-L\right)\right)=0 \text { as per Eq. } \\
\text { (1). }\end{array}$ \\
\hline
\end{tabular}


ii. We have shown that a slight shift of the time lag can change the type of coupling on the neutral curve. The regions of the neutral curve close to the intersection of the neutral curve segments $\omega_{c}^{i} \tau=0$ and $\omega_{c}^{i} \tau=\pi$, as well as, $\omega_{c}^{i} \tau=0$ and $\omega_{c}^{i} \tau=-\pi$, manifest strong evidence of coupling. Near these intersections, for each value of $\tau$ we find two sets of solutions for $n$ -threshold. As a rule, the larger values of $n$-thresholds correspond to the coupled mode (BI) and the smaller values correspond to the coupled mode (BA). Thus, for a weak flame with low interaction index, even though the intrinsic mode does not become unstable on its own, it might trigger instability of one of the nearby acoustic modes.

iii. We have verified our analytical model by numerics and the accuracy of our analytical result is found to be good. The accuracy of prediction is very good as we stay close to the neutral curve, which is the area of most interest and where the actual intrinsic-acoustic mode coupling takes place. The quartic equation model (14) provides excellent accuracy not only in the vicinity of the neutral curve, but away from it also. It can be easily shown that the existence intrinsic-acoustic mode coupling in practical combustors is robust and not affected by the temperature jump across the flame.

In our present analysis, we have confined our attention to the linear coupling within the framework of a 1-D model of a quarter-wave resonator with the $n-\tau$ model of flame heat release. However, we expect the phenomenon of coupling to occur in reality under a very broad range of conditions. For the sake of simplicity, here we considered only a quarter wave resonator with ideal end conditions. Similar analysis was carried out for the ideal open-open system and the same four types of coupling were also found (Mukherjee, 2017, 2018). The coupling in combustors with non-ideal boundary conditions needs a dedicated investigation. Our current work is based on the assumption of the dominance of velocity coupling, i.e. upstream velocity perturbation leads to flame heat release perturbation. What happens under different coupling scenarios (i.e. pressure or equivalence ratio coupling), will call for further investigation. If we also introduce nonlinearity in the system, the simplest $n-\tau$ framework for understanding flame response leads to an amplitude-dependent $\tau$ value (Lieuwen, 2012). A dedicated study of non-linear mode coupling lies in the scope of future work, as well.

\section{Acknowledgements}

The work is a part of the Marie Curie Initial Training Network Thermo-acoustic and aeroacoustic nonlinearities in green combustors with orifice structures (TANGO). We gratefully acknowledge the financial support from the European Commission under call FP7-PEOPLEITN-2012.

\section{References}

Bauerheim, M., Nicoud, F., and Poinsot, T. 2015. Theoretical analysis of the mass balance equation through a flame at zero and non-zero Mach numbers. Combust. Flame, 162, 6067.

Craik, A. 1985. Wave interactions and fluid flows. Cambridge University Press. 
Crocco, L., and Cheng, S. 1956. Theory of combustion instability in liquid propellant rocket motors. Butterworths Scientific Publications.

Dowling, A. 1995. The calculation of thermo-acoustic oscillations. J. Sound Vib., 180(4), 557581.

Emmert, T., Bomberg, S., Jaensch, S., and Polifke, W. 2016. Acoustic and intrinsic thermoacoustic modes of a premixed combustor. Proc. Combust. Inst., 36, 3835-3842.

Hoeijmakers, M., Kornilov, V., Arteaga, I., Goey, P., and Nijmeijer, H. 2014. Intrinsic instability of flame-acoustic coupling. Combust. Flame, 161, 2860-2867

Lieuwen, T. 2003. Modelling premixed combustion-acoustic wave interactions: a review. $J$. Propul. Power, 19(5), 765-781

Lieuwen, T., and Yang, V. 2005. Combustion instabilities in gas turbine engines: Operational experience, fundamental mechanisms, and modelling. Prog. Astronaut. Aeronaut., AIAA.

Lieuwen, T. 2012. Unsteady combustor physics. Cambridge University Press.

Magina, N., Acharya, V., and Lieuwen, T. 2019. Forced response of laminar non-premixed jet flames. Prog. Energy Combust. Sci., 70, 89-118.

Mukherjee, NK. 2017. Analytic study of intrinsic flame and coupled-intrinsic acoustic instabilities in combustor models. Ph.D. thesis, Keele University.

Mukherjee, NK., and Shrira, V. 2017. Intrinsic flame instabilities in combustors: Analytic description of a 1-D resonator model. Combust. Flame, 185C, 188-209.

Mukherjee, NK. 2018. Analytic description of flame intrinsic instability in one-dimensional model of open-open combustors with ideal and non-ideal end boundaries. Int. J. Spray Combust., 10(4), 287-314.

Munjal, ML. 1987. Acoustics of Ducts and Mufflers. John Wiley, New York.

Polifke, W., Paschereit, C., and Dobbeling, K. 2001. Constructive and destructive interference of acoustic and entropy waves in a premixed combustor with a choked exit. Int. J. Acoust. Vib., 6(3), 135-146.

Truffin, K., and Poinsot, T. 2005. Comparison and extension of methods for acoustic identification of burners. Combust. Flame, 142, 388-400.

Vishnu, R., Sujith, R., and Aghalayam, P. 2015. Role of flame dynamics on the bifurcation characteristics of a ducted V-flame. Combust. Sci. Technol., 187, 894-905. 


\section{Appendix A}

\section{A.1. Pressure profiles of acoustic and intrinsic modes for pure acoustic and pure intrinsic instability}

Here we analyze the pressure profiles of acoustic and intrinsic modes when we do not have any coupling of modes, and thus, we have pure acoustic and pure intrinsic instability. Then we discuss what the mode shapes might reveal about identities of the modes under consideration.

Figs. A.1 (a, b, c, d) illustrate a scenario of conventional "pure" acoustic mode instability for a fixed time lag ( $\tau=4.5 \mathrm{~ms}$ ), where the first acoustic mode becomes unstable. The pressure profile of the chosen acoustic mode behaves in a manner typical of the first acoustic mode of a closed-open combustor. The decaying intrinsic mode for $n=0.3$, as shown in Fig. A.1 (a), has a sharp hump (at the flame location $x_{q}=L / 2$ ) in the pressure profile. As $n$ is increased from 0.3 to 5.0 (shown in Figs. A.1 (c, d)), we cannot spot any qualitative change in the pressure profile of the intrinsic mode. Thus, in the absence of coupling, there is a clear distinction between the mode shapes of acoustic and intrinsic modes for this case, where pure acoustic mode instability prevails.

Similarly, in Figs. A.1 (e, f, g, h) we illustrate pure intrinsic mode instability. The same scenario is also depicted in $\S 6$ (Fig. 20) of Mukherjee and Shrira (2017). As per Fig. A.1 (e), at $\tau=2.2 \mathrm{~ms}, n=0.4$, both the acoustic and intrinsic modes decay on the complex frequency plane. The pressure profile of acoustic mode is typical of the classical first acoustic mode of a closed-open combustor, whereas, for the intrinsic mode, the pressure profile manifests a prominent dip at the flame location $x_{q}=L / 2$. As we increase $n$ to 1.1 , the intrinsic mode becomes unstable, whereas, the acoustic mode decays. Fig. A.1 (g) represents this case. The figure shows that the acoustic mode retains profile visually identical to that of classical first acoustic mode for a closed-open combustor, but, the dip in the intrinsic mode pressure profile becomes more prominent.

Thus, whenever pure intrinsic mode instability exists we can clearly distinguish the mode shapes of acoustic and intrinsic modes in such a scenario. 


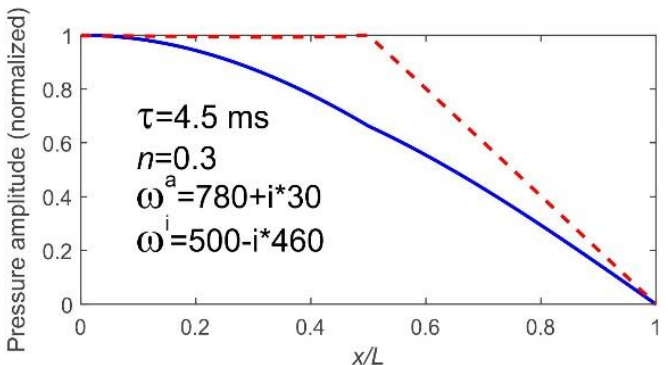

(a)

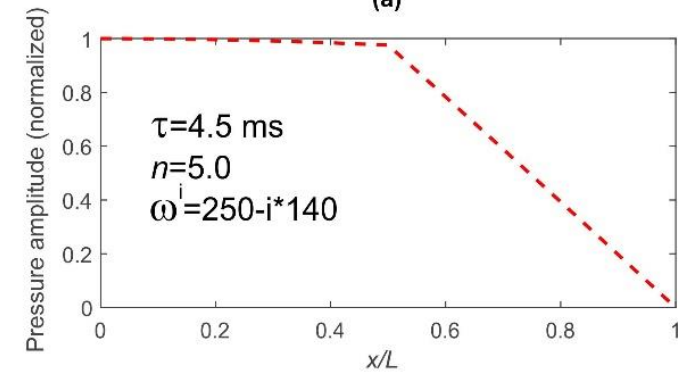

(c)

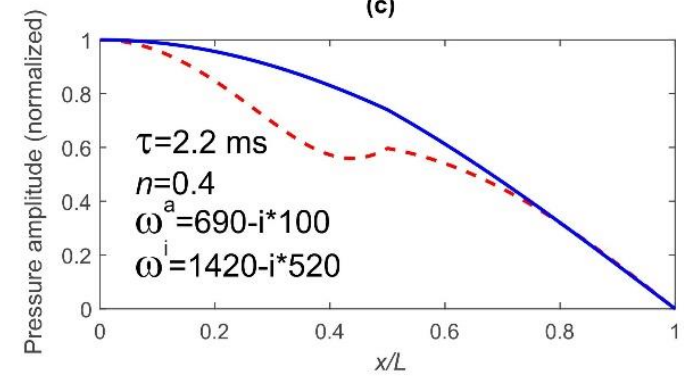

(e)

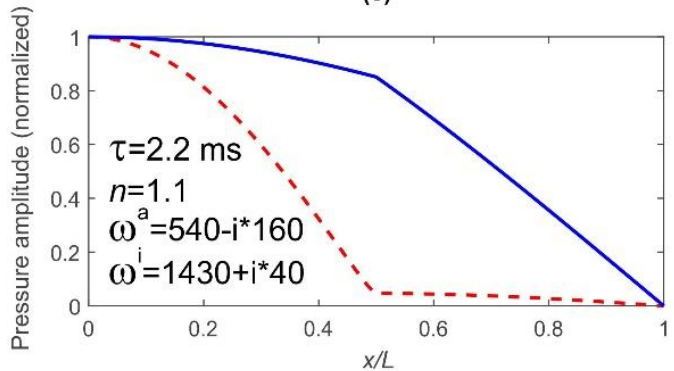

(g)

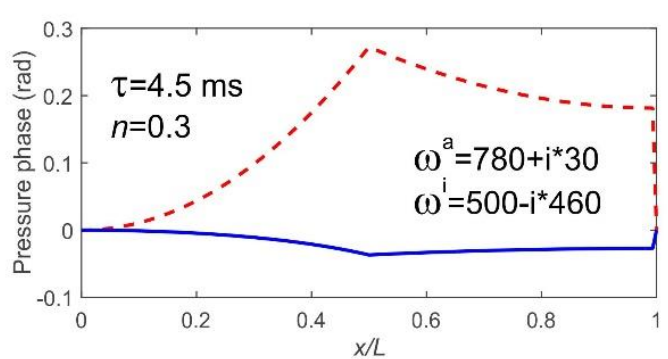

(b)

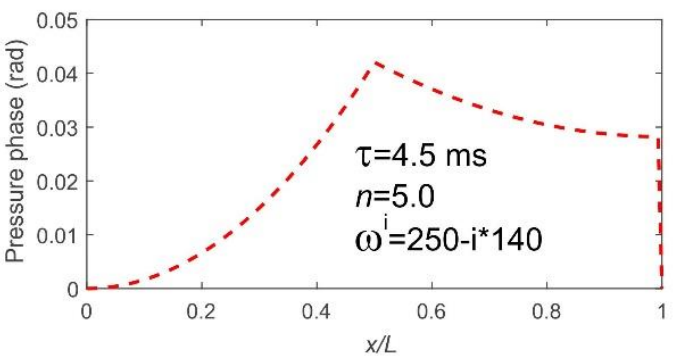

(d)

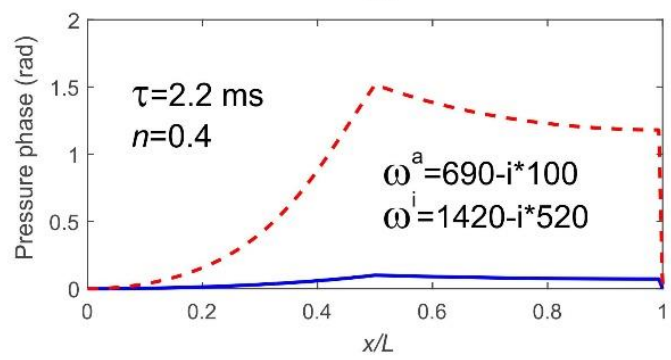

(f)

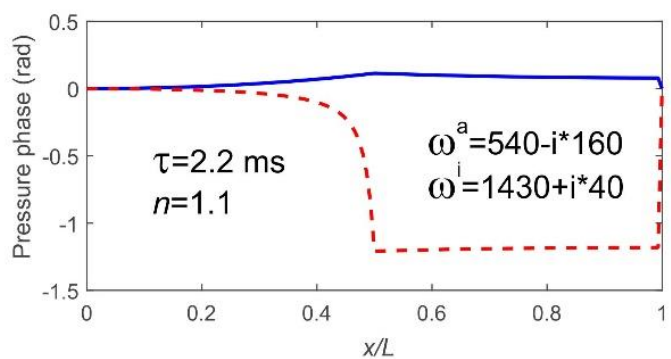

(h)

Figure A.1: Normalized pressure amplitude and phase in radians of pure intrinsic and acoustic modes given by Eqs. (10) and (12), for $x_{q}=L / 2$. The left and right columns of the figure panels represent the pressure amplitude and phase, respectively. The cross-section and temperature are assumed to be uniform. The dashed (red online) and solid (blue online) lines correspond to the intrinsic and acoustic modes, respectively. 